\title{
Pêcher pour survivre dans le Rufiji
}

Sources orales et interprétations

Jean-Luc Paul

\section{(2) OpenEdition}

Journals

Édition électronique

URL : https://journals.openedition.org/eastafrica/300

DOI : 10.4000/eastafrica.300

ISSN : 2790-1076

Éditeur

IFRA - Institut Français de Recherche en Afrique

Édition imprimée

Date de publication : 1 mai 2015

Pagination : 113-154

ISSN : 2071-7245

Référence électronique

Jean-Luc Paul, «Pêcher pour survivre dans le Rufiji », Les Cahiers d'Afrique de l'Est / The East African Review [En ligne], 50 | 2015, mis en ligne le 07 mai 2019, consulté le 11 octobre 2022. URL : http:// journals.openedition.org/eastafrica/300; DOI : https://doi.org/10.4000/eastafrica.300 


\title{
Pêcher pour survivre dans le Rufiji, Sources orales et interprétations
}

\author{
Jean-Luc Paul ${ }^{1}$
}

En 2011, nous avons publié un article (Paul et al., 2011) qui traitait des pratiques de braconnage halieutique dans la plaine inondable du fleuve Rufiji (Tanzanie). Les habitants de cette plaine vivent d'une économie paysanne où se combinent la production d'autoconsommation et la production marchande. La production d'autoconsommation est essentiellement agricole (riz, maïs...) tandis que la nature de la production marchande est diversifiée. Elle varie notamment en fonction de la localisation géographique (degré d'éloignement des zones urbaines, isohyète, distance au fleuve...). Dans le tiers occidental de la plaine inondable, la pêche est la seule activité marchande accessible à tous les foyers. C'est en général une pêche au filet qui se pratique à partir de pirogues monoxyles dans les lacs qui ourlent le fleuve et à proximité desquels sont installés les villages. Les revenus qu'on en tire sont modestes, en moyenne 1,20 € par jour de pêche en 2009. Ils permettent, bon an mal an, de couvrir les besoins monétaire basiques des foyers. Mais, dès qu'un besoin plus important apparaît, le précaire équilibre des finances domestiques est rompu. L'article cité évoque les études secondaires d'un adolescent dont le financement entraine le doublement des besoins monétaires de sa famille. Pour les couvrir, le père du collégien n'aura d'autre choix que d'abandonner la pêche de proximité et de se rendre dans les lacs poissonneux de la réserve du Selous. Il rentre ainsi, contraint et forcé, dans l'illégalité et s'expose à plusieurs dangers mortels : attaques d'hippopotames ou de crocodiles, tirs des gardes-chasse... L'article démontre qu'il n'existe pas d'alternative à cette stratégie. Cette démonstration mobilise un corpus de données de plusieurs natures. Il s'agit de données quantitatives issues du suivi quotidien, durant trois ans, d'un échantillon de pêcheurs, d'observations directes et, enfin, de témoignages de villageois. Si l'article propose des extraits de ces témoignages, traduits en français, son format ne se prête pas à la restitution in extenso du corpus discursif non plus qu'à la simple présentation de l'original swahili des extraits utilisés.

La présente publication, à l'image d'une précédente publication (Paul, 2014), livre ce corpus dans la langue originale de recueil et son interprétation française. Son volume rend cependant difficile sa publication intégrale et seule une large sélection est ici présentée ${ }^{2}$. Après un chapeau introductif décrivant les conditions de recueil des témoignages et les caractéristiques générales de la pêche dans le Selous, les témoignages sont présentés. Quand

\footnotetext{
${ }^{1}$ Anthropologue, UMR 8171 IMAF (Institut des Mondes Africains). jlpaul@univ-ag.fr

${ }^{2}$ Soit environ un tiers du corpus total des enquêtes portant spécifiquement sur le braconnage halieutique.
} 
Les Cahiers d'Afrique de l'Est

elle mobilise une connaissance approfondie de spécificités locales, l'interprétation est enrichie de notes de bas de page. Enfin, dans le texte en swahili, les mots en italiques appartiennent à l'idiome du Rufiji.

\section{Les conditions de recueil des récits}

J'ai vécu deux ans, d'octobre 2006 à octobre 2008, dans le village de Kipo, sur le bord du fleuve Rufiji. J'estime ma présence physique dans le village à six mois la première année et neuf mois la seconde. J'ai ensuite effectué des séjours d'un mois en 2009, 2010, 2012 et 2013. Je vivais chez l'habitant. J'ai lié de solides relations de camaraderie et d'amitié et la confiance acquise m'a permis d'aborder avec mes interlocuteurs les sujets les plus délicats. Le projet employait un technicien, Kassim Kindinda, villageois de Kipo ayant étudié jusqu'en form 4, soit 1'équivalent de la seconde. Agriculteur et pêcheur comme pratiquement tous les villageois, il m'a aidé à identifier les meilleurs informateurs. Nous avons conduit les entretiens en commun, directement en swahili et donc sans traducteur. Ils se déroulaient à proximité de 1'habitation du narrateur. Nous étions la plupart du temps entourés de quelques amis ou curieux. Les entretiens ont été enregistrés puis nous les avons saisis sur un logiciel de traitement de texte, à nouveau conjointement. J'ai ensuite assuré seul l'interprétation française. Kassim Kindinda ne parle pas français et son anglais est limité. L'interprétation française a été soumise pour vérification à des collègues maîtrisant le swahili et le français ${ }^{3}$.

\section{Les caractéristiques générales de la pêche dans le Selous}

La demande en poisson fumé des classes populaires citadines est importante. L'approvisionnement est assuré par des intermédiaires qui achètent le poisson aux pêcheurs de diverses zones rurales de Tanzanie et l'acheminent, fumés, vers les villes, Dar-es-Salaam principalement. Ces intermédiaires possèdent un capital limité, ils ne disposent pas de véhicules et empruntent les bus assurant les liaisons régulières entre la ville et les villages pour transporter leur marchandise. Leur capital limité explique, d'une part, la nécessité d'un turn-over rapide afin de dégager des revenus décents et, d'autre part, le fait qu'une zone de pêche donnée est toujours livrée à la concurrence de plusieurs intermédiaires. L'offre de poisson excédant rarement la demande, les pêcheurs disposent d'une certaine marge de négociation.

Les pêcheurs, quant à eux, disposent d'une faible trésorerie. En général, le revenu de la pêche quotidienne dans les lacs de proximité est utilisé immédiatement. Cette pêche légale est en effet peu productive. La pêche dans les lacs distants de la réserve du Selous permet un

\footnotetext{
${ }^{3}$ Je remercie Ida Hadjivayanis de la SOAS (School of Oriental and African Studies) et Salha Hamdani de leur relecture attentive du texte.
} 
triplement du revenu mais elle entraîne l'éloignement du pêcheur durant une huitaine de jours en moyenne. Durant cette période, il est dans l'incapacité de fournir à sa famille la somme quotidienne nécessaire à sa survie. L’intermédiaire, intéressé par un approvisionnement conséquent de poissons de qualité, se transforme alors, en quelque sorte, en armateur et préfinance l'expédition. Il avance la somme nécessaire à l'équipement du pêcheur et au fonctionnement de son foyer durant son absence.

Tableau de préfinancement d'une expédition de pêche au Selous en 2009

\begin{tabular}{|c|c|c|c|c|c|c|c|}
\hline Item & Filet & $\begin{array}{c}\text { Location de } \\
\text { pirogue } \\
\text { plastique }\end{array}$ & $\begin{array}{c}\text { Bâche } \\
\text { et sel }\end{array}$ & Divers & $\begin{array}{c}\text { Total avance } \\
\text { monétaire aux } \\
2 \text { familles }\end{array}$ & Total \\
\hline $\begin{array}{c}\text { Coût } \\
\text { (euros) }\end{array}$ & 6 & 6 & 1,8 & 4,95 & 2,28 & 12 & $\mathbf{3 3 , 0 3}$ \\
\hline
\end{tabular}

Le braconnage dans les lacs du Selous est très contraint. Le plus souvent plusieurs pirogues, chacune d'entre elles accueillant deux pêcheurs, s'associent en une même expédition à fin d'entraide. Pour échapper aux gardes, elles remontent nuitamment le fleuve jusqu'à l'un des lacs du delta intérieur. La pêche et le fumage eux aussi seront nocturnes. Pêchant dans la pénombre, les pêcheurs s'exposent à l'irascibilité des hippopotames et à la voracité des crocodiles avides des poissons pris dans les filets. Durant la journée, leur repos est perturbé par les visites des hyènes attirées par l'odeur du poisson ou par le passage des patrouilles terrestres, fluviales ou plus rarement aériennes des gardes.

Le déroulement d'une expédition de pêche pour le Selous à partir du village de Kipo est le suivant :

- Jour 1 : se rendre en bus à Mloka, le village frontalier de la réserve, y rencontrer l'intermédiaire, effectuer les achats nécessaires à l'expédition (filet, provision), louer une pirogue et confier à une personne de confiance l'argent à remettre à la famille.

- Nuit 1 : remonter le fleuve jusqu'au lac choisi et installer un bivouac à couvert ${ }^{4}$.

- Nuits 2 à 6 : pêcher (et fumer progressivement le poisson) $)^{5}$.

- Nuit 7 : Descendre le fleuve jusqu'à Mloka.

- Jour 8 : Vendre le poisson à l'intermédiaire. Le retour à Kipo se fait en général le lendemain matin.

\section{Témoignage 1}

\footnotetext{
${ }^{4}$ Pour un lac très éloigné comme Suwanda, il faut compter deux nuits de voyage.

${ }^{5}$ Le nombre de jours de pêche est fixé par la capacité de transport de la pirogue.
} 
La richesse et les difficultés de la collecte de témoignages sont bien illustrées par le récit de Maurice ${ }^{6}$. Mo, comme nous l'appelons au village, décrit précisément les étapes d'une expédition de pêche particulière qui se déroule sans encombre jusqu'à la rencontre avec un hippopotame irascible, sur le chemin du retour. Le swahili de Mo est souvent grammaticalement fautif. Sa manière de raconter est confuse et hésitante. Au village, Mo est mon voisin c'est ici sa manière de parler à laquelle je me suis accoutumé. Déjà laborieuse en swahili, la lecture du récit en français, s'il était traduit " au plus près », deviendrait rébarbative. Le texte français est donc ici une interprétation qui contourne en partie les piètres qualités de conteur de Mo.

\section{Témoignages 2 à 4}

Le swahili des trois témoignages suivants est plus fluide. Ils racontent des expéditions surprises par des gardes-chasse. Ils abordent les relations entre les pêcheurs, les relations entre pêcheurs et armateurs et dressent une sorte de typologie des lacs du Selous en fonction de la productivité de la pêche, de la dangerosité et de l'accessibilité.

\section{Témoignage 1, le 21 décembre 2007}

A vrai dire, je serais incapable de me souvenir du moment où ça s'est passé. Nous sommes partis d'ici, moi et Eugène, mon cadet. Nous avons pris notre pirogue et nous sommes montés à Mloka. Une fois arrivés à Mloka, nous avons organisé notre travail avec notre armateur. Il s'appelait Ignace. Il nous a donné de l'argent. Nous sommes allés dans la réserve du Selous, moi et Eugène. Il y avait aussi Serge et Thomas, Roger et Jacques. Nous étions six personnes pour trois pirogues. Nous sommes sortis de là jusque dans la brousse, par là-bas, dans le Selous. En pénétrant dans le Selous, nous nous sommes rendus tout d'abord au lac Manze, tout à fait au bout du lac. Georges nous a dit :

- Par ici nous n'attraperons pas beaucoup de poissons, retournons en arrière, allons près de l'entrée du $l a c^{7}$.

On est parti pour l'entrée du lac. On a pêché. Chacune des trois pirogues a pêché beaucoup de poissons. On a redescendu le fleuve. Même le jour où nous avons descendu le fleuve, j'ai oublié quel jour c'était. Alors que nous descendions au sortir de la brousse, làbas... on descendait, descendait le fleuve. On a dépassé Vinangu, on a dépassé Mtemele. C'est-à-dire que nous étions tout près d'atteindre le village de Mloka, nous avions franchi tous les obstacles et tout allait bien.

\footnotetext{
${ }^{6}$ Tous les noms ont été changés pour interdire l'identification des pêcheurs.

${ }^{7}$ Là où le chenal de connexion avec le fleuve débouche.
} 
Kwa kweli ile siku sitaweza kuikumbuka ilikuwa siku gani. Tulitoka hapa, mimi na bwana mdogo wangu anaitwa Eugène. Tukachukua ngalawa yetu hapa sisi. Tukapandisha mpaka Mloka. Tulivyofika Mloka, tukapanga kazi kwa tajiri. Tajiri wetu alikuwa anaitwa mzee Ignace. Akatugea hela. Tukaenda Selous. Tulikuwa mimi na Eugène. Akawa Serge na Thomas. Akawa Roger na Jacques. Tukawa watu sita kwa ngalawa tatu. Tukatoka hapa sisi mpaka porini. Huko Selous tukaenda. Tulivyoingia kwanza tulienda Manze yenyewe, tukaingia ndani kabisa. Georges akatuambia :

- Huku samaki wenyewe hatuwezi kupata kwa wingi, turudi. Twendeni kule nje.

Tukatoka tukaja karibu ya nje. Tukawa tunavua. Tukavua samakiiiiii... Tukapata samaki wengi katika ngalawa zetu zote tatu. Tukawa tunashuka. Mpaka ile siku ya kushuka nayo nimesahau ile siku. Sasa tulivyoshuka kutoka na huku porini... Tukashuka sisiii, yaani Vinangu kote tukapita, Mtemele tukapita. Yaani karibu kabisa ya kutokea huku kijijini. Yaani mipaka yote tumeshaivuka, bado tupo.

Avant d'arriver au village, il y a un bras du fleuve qu'on appelle Vumbi. Ce bras ce jette dans le fleuve au niveau du poste de garde. Comme nous arrivions là, avant de pénétrer dans le fleuve, les trois pirogues se suivaient. La pirogue de tête, c'était celle de Georges. Je suivais et ensuite venait Roger. Roger a coupé, il est passé sur le côté, sur l'autre rive. Ensuite, je suis passé devant Serge et je suivais Georges, là-bas devant, où il était. Comme je coupais pour aller sur l'autre rive, un hippopotame a surgi. Au moment où l'hippopotame est sorti, la manière dont il venait, je n'avais plus la force de pagayer. Je l'écoutais en me demandant ce qu'il allait faire. Il arrivait la gueule grande ouverte. Il a frappé l'avant de la pirogue où se trouvait mon cadet Eugène. Eugène est tombé à l'eau et moi je suis resté dans la pirogue. Comme j'étais encore à bord de la pirogue, l'hippopotame est revenu. J'avais saisi ma pagaie et j'ai pagayé pour conduire ma pirogue sur le côté. L'hippopotame s'est dit :

- Tiens ! Il y a encore quelqu'un à bord !

Il revenait, il revenait la gueule grande ouverte. A cette période la lune brillait beaucoup. D'une rive à l'autre du fleuve tu voyais clairement. Et moi est-ce que je n'étais pas en train de pagayer alors que l'hippopotame revenait? 
Sasa kabla hatujafika kijijini kuna mto mmoja, unaitwa mto wa Vumbi. Huo mto ndiyo unaungana na mto mkubwa kabisa, kuja kutokea forodhani. Tulivyotoka kule sisi, kabla hatujaupata mto mkubwa, ndiyo tukawa sasa hapo tunafukuzana ngalawa tatu. Ngalawa ya kwanza ilitangulia ngalawa ya Georges. Nikafuata mimi akaja Roger. Roger yeye akakata, akapita pembeni, akapita ng'ambo ya pili. Serge akawa nyuma yangu mimi. Mimi nikawa mbele. Sasa mimi nikawa 'namfuata Georges kule mbele alipokuwepo. Nilipokata kutoka pembeni ya ng'ambo, kwenda pembeni ya ng'ambo nyingine ndiyo akatoka 'boko. Alipotoka 'boko, alivyokuja yule 'boko, mimi pale nikawa sina nguvu ya kuvusha. Nilikuwa namsikiliza atafanya nini ?! Alipokuja yule 'boko, akawa anakuja mdomo wazi. Akaja akapiga mbele kule kwa bwana mdogo yule Eugène. Eugène ikabidi akatumbukia kwenye maji. Alipotumbukia kwenye maji, nikabakia mimi kwenye ngalawa. Kubakia kwenye ngalawa, yule 'boko anarudi, mimi 'nashika 'bao navusha ile ngalawa kusudi 'naenda pembeni. Yule 'boko akaona :

- Halaaa ! Kumbe mle kuna mtu mwengine nimemwacha.

Akawa anakuja. Anakuja mdomo wazi kabisa. Kipindi hicho lakini mwezi sasa ndiyo unawaka sana. Unaweza kuona mpaka ng’ambo kabisa kuwa kile kitu gani na kile kitu gani. Sasa si mimi ninavyovusha na 'boko anapokuja?

Finalement il a fendu la pirogue par-dessous et moi je suis tombé à l'eau. Mais par chance, quand je suis tombé à l'eau, la pirogue est restée en place bien qu'elle ait été endommagée. Comme elle était fendue, l'eau rentrait par-dessous. Moi je suis tombé à l'eau et j'avais de l'eau jusqu'au cou. Qu'est-ce que je devais faire ? J'ai pataugé en allant là où l'eau était moins profonde. Mais il me suivait. Comme il me suivait, la première chose que j'ai faite c'est de plonger et d'avancer sous l'eau. J'ai émergé plus loin. Comme j'émergeais, Serge venait derrière moi. Il avait vu toute la scène. Serge et Thomas n'avaient pas d'autre chose à faire que de pagayer pour aller sur le côté. Serge a dit à Thomas :

- Eh ! Pour l'instant ne pagaie pas. Si tu pagaies il va nous suivre nous.

Serge et Thomas se sont tenus tranquilles. L'hippopotame était après nous. Serge a dit :

- Maintenant déplaçons-nous.

Serge et lui se sont déplacés du côté de la berge d'où nous venions. Ils sont retournés. Moi et Eugène on fuyait vers l'autre berge. 
Ikabidi aipasue chini, chini kukawa kumepasuka. Ikabidi mimi nitumbukie kwenye maji. Lakini ile ngalawa kwa bahati nzuri haikupinduka tangu ilipokaa vile vile, lakini ilipatwa na msukosuko. Tangu ilipoingia maji na chini ilipotoboka kule ikawa inaingia maji. Mimi nilipotumbukia kwenye maji, maji mimi yakawa ya shingo. Ikawa mimi sasa "nafanya nini? Nikawa 'nakubuga, 'nafuata maji madogo. Yule 'boko akaona :

- Halaa! Kumbe kuna mtu mwengine anakubuga.

Sasa ananifuata mimi. Aliponifuata mimi, mimi cha msingi nikafanya pole nikazama fumbo, yaani nikazama katika maji. Nilipozama katika maji, nikawa 'natembea chini kwa chini. Kufika mbele nikaibuka. Kuibuka ndiyo nyuma akawa Serge anakuja. Yaani huyu Serge hili tukio lote yeye analiona bayana kabisa. Serge akawa hana tena jinsi yakufanya, ikabidi yule Thomas naye ikawa anavusha kusudi aende pembeni. Serge akamwambia :

- Wee ! Usivushe kwanza. Kwa sababu ukivusha huyo anaweza kutufuata sisi.

Serge, huyu Thomas ikabidi atulie. Yule 'boko akawa anatufuata sisi. Serge akasema :

- Sasa hapa sasa na sisi tusogee.

Serge naye ikabidi asogee pembeni ya kule ng'ambo tulipotoka. Akarudi Serge. Mimi na Eugène tukawa tunakimbia upande wa pili, tulipokuwa tunakwenda sisi.

L'hippopotame est revenu en arrière. Moi j'ai atteint la terre ferme. J'ai atteint la terre ferme et Eugène aussi. On est restés sur le côté. On avait perdu nos pagaies, nos vêtements étaient trempés et tout était perdu. Ce qui nous restait à faire, dire à Serge et les autres qui étaient sur l'autre berge :

- Les amis, cherchez notre pirogue par-là !

Serge et Thomas ont vu la pirogue et l'ont saisie. Ensuite ils ont traversé depuis la berge où ils s'étaient réfugiés jusqu'à l'autre bord où nous nous trouvions. Ils menèrent notre pirogue jusqu'à l'endroit où nous nous trouvions. Arrivés là, il n'y avait pas le choix. Ils nous ont dit de vider l'eau de la pirogue. On a pris une casserole pour écoper. La pirogue ne s'était pas renversée, elle était restée d'aplomb. Moi j'écopais et je voyais que ça n'en finissait pas. Chaque fois que j'écopais, il y avait encore de l'eau. Je leur ai dit :

- Vous savez, cette pirogue elle est fendue parce que j'ai beau écoper, il y a toujours de l'eau.

Ils m'ont dit que ce n'était pas possible. Je leur ai dit :

- Attendez que j'inspecte la pirogue. 
Sasa tulichofanya pale, yule 'boko aliyegonga ule mtumbwi na aliyekuwa ananifuata mimi, mimi ikabidi pale nizame fumbona kuondoka. 'boko ikabidi akawa anarudi nyuma. Sasa akawa anarudi nyuma. Mimi pale nikageuka gongoni. Nikageuka gongoni mimi na Eugène. Tukakaa pembeni. Tukakaa pale tena 'bao zetu zote zimeshapotea, nguo zetu zote zimeshalowa kila kitu kikawa kimeshapotea. Pale cha kufanya tukawaambia kina Serge wakiwa ng'ambo ya pili :

- Jamani angalieni ngalawa yetu huko!

Serge na Thomas ikabidi wakaiona ile ngalawa wakaikamata ile ngalawa. Na wakavusha kutoka kule ng'ambo walipokimbia kutufuata huku ng'ambo tulipo sisi. Wakaleta ngalawa yetu mpaka kule pembeni tulipofika. Kufika kule tukawa hatuna jinsi. Wakasema sasa kupeni ngalawa hiyo. Tukawa tumechukua sufuria tukawa tunakupa maji katika ngalawa. Ile ngalawa lakini ilikuwa haikupinduka ilikuwa imekaa vilevile. Sasa mimi kila nikitoa maji 'naona maji hayaishi. Kila nikitoa maji, maji hayaishi. Nikawaambia :

- Ujue hii ngalawa hii imeshapasuka kwa sababu gani ? Mimi kila nikitoa maji, maji hayatoki.

Wakaniambia :

- Hapana. Hivyo ngalawa haijapasuka.

Nikawaambia :

- Hapana ngojeni kwanza mimi niiangalie hii ngalawa.

Ma torche électrique, je l'avais laissée dans la pirogue, contre le poisson, et elle n'était pas perdue. Je me suis souvenu que j'avais laissé ma torche sur le côté, dans la pirogue. J'ai tâtonné et je l'ai prise. Quand j'ai voulu l'allumer, elle n'avait pas de puissance parce qu'elle avait pris l'eau. Je ne sais pas quel était le problème mais elle ne produisait pas de lumière forte. Et j'étais le seul de l'expédition à avoir une torche qui avait un fort éclairage. Alors j'ai passé mon bras dessous la pirogue et j'ai tâtonné. En tâtonnant, j'ai rencontré l'endroit où la dent de l'hippopotame était entrée. Mon pouce pénétrait dans le trou. Je leur ai dit que la pirogue était percée par-dessous. Georges est venu, il a inspecté et il a dit que c'était vrai, la pirogue était percée. Que devions-nous faire ? Rien d'autre que de renverser la pirogue sur la terre ferme et décider de ce qu'il y avait à faire. Tous ensembles, on a retourné la pirogue sur 
la rive. On a sorti tout le poisson, il avait pris l'eau et il était en pièces, il n'y avait plus rien à en tirer. On a sorti le poisson et on l'a mis sur le côté. Ensuite on a renversé la pirogue pour voir comment c'était dessous. Comme les torches n'avaient pas de puissance, on a éclairé tant bien que mal. Georges a dit :

- Eh bien ! Il va falloir chercher du bois pour y mettre le feu, sans ça vous ne pourrez jamais partir.

Tochi yangu ikawa nimeiweka mle mle pembeni mwa samaki, ikawa sasa haijapotea. Nikakumbuka kuwa tochi yangu niliweka pembeni mwa ngalawa. Nikapapasia, nikachukuwa tochi yangu. Ile tochi nilivyowasha ikawa sasa haina nguvu kwa sababu ilishaingia maji. Sijui ilikuwa ina hasara gani tena ikawa haina uwezo wa kutoa mwanga mkali. Na wote kwenye safari yetu mwenye tochi yenye kutoa mwanga mkali nilikuwa mimi peke yangu ilikuwa na mwanga mkali. Sasa nikatia mkono kwa chini, nikawa 'napapasa, kupapasa nikakuta sehemu kuna jino la boko limeingia. Nilipotia kidole ikawa dole langu gumba hili likawa limezama pale sehemu, nikawaambia :

- Hii ngalawa chini imeshatoboka.

Akaja Georges akaiangalia akasema :

- Kweli ngalawa imeshatoboka. Sasa tufanye nini ? Hamna cha kufanya, tugeuze ngalawa gongoni. Tuangalie jinsi ya kufanya wote pale tukashirikiana kwa pamoja.

Tukatoa wale samaki, halafu tukaigeuza ile ngalawa kwa kuiangalia kule chini kupoje ? Kwa kuwa tochi zenyewe zilikuwa hazina mwanga mkali tukamulika hivyo hivyo. Georges akasema :

- Halaa ! Mpaka sasa hivi inabidi tutafute manjengo ili tuchomee. Bila hivyo nyie mkisema kuondoka mtashindwa.

Et c'est vrai que les pirogues de nos comparses étaient déjà pleines. Le poisson ne laissait pas la place à quelqu'un pour s'asseoir, à moins d'en ôter une partie. Alors j'ai retiré mon pantalon et j'ai déchiré l'une de ses jambes. Je l'ai découpé en lambeaux. Une jambe de pantalon déchirée. Ensuite j'ai commencé à l'insérer là où c'était percé. On a calfeutré et calfeutré encore et ça ne suffisait pas. J'ai déchiré la seconde jambe et on a continué à calfeutrer. Et comme ça jusqu'à ce qu'il n'y en ait plus, il n’y avait plus de tissu. Je portais 
Les Cahiers d'Afrique de l'Est

une chemise à manches longues. J'ai déchiré une manche pour calfeutrer la pirogue encore jusqu'à ce que ça soit à peu près satisfaisant. On l'a remise à l'eau. Elle prenait encore l'eau, mais pas beaucoup. Elle prenait encore l'eau mais lentement. On s'est dit que c'était suffisant. On a pris le poisson et on l'a mis dans la pirogue. En fait ce n'était plus du poisson, c'était de la chiquetaille ! On l'a mise dans la pirogue. On avait perdu nos deux pagaies. Ils nous ont donné une pagaie. Georges nous a donné une pagaie. Chaque pirogue avait deux pagaies. Eux, ils nous ont donné une pagaie et Georges est resté avec une pagaie.

Ile ngalawa tukaigeusha gongoni. Tukatoa samaki wote, walioloaloa, wote wakavunjikavunjika samaki, ikawa hamna tena hamu ya samaki, tukawatoa tukaweka pembeni.

Na kweli, ngalawa za wenzetu zote zilikuwa zimeshajaa. Samaki zikawa tena hamna tena nafasi nyingine ya kukaa labda wapunguze samaki apate kukaa mtu. Sasa pale tulichokifanya pale mimi nilivaa suruali. Nikakata suruali yangu mguu mmoja. Nikaukata kama vile pensi. Mguu mmoja pale chaaa! Nilivyoukata ule, nikawa sasa nikaanza kuchomea kulipopasuka. Tukachomeachomea... Nikawa bado naendelea. Nikakata mguu wa pili. Tukachomea hivyo hivyo. Hivyo hivyo mpaka ikaisha yote, ikawa sasa pale hamna matambaa mengine, nilivaa shati la mikono mirefu, nikakata mikono mirefu nikachomea ile ngalawa mpaka ikawa imesimama kidogo. Tukaitia kwenye maji. Ikawa inatoa maji lakini siyo mengi. Ilikuwa inatoa maji siyo ya nguvu kweli. Tukasema hapa inatosha. Tukachukua samaki. Tukawaweka katika ngalawa. Wale samaki walikuwa siyo samaki tena, ilikuwa makasamala. Tukawatia kwenye ngalawa yetu mle. 'bao zote sisi zimeshapotea. Wakatupa 'bao moja. Akatupa Georges. Katoa moja, akasema :

- Chukueni kafu.

Yeye alikuwa na kafi mbili. Ila kila ngalawa ilikuwa na kafi mbili mbili. Sasa wao wakatupa kafi moja. Georges akabakiwa na kafi moja.

Il n’y avait plus qu'une personne qui pagayait. Moi je pagayais et Eugène lui écopait l'eau qui rentrait doucement. On a pagayé depuis l'endroit où avait eu lieu l'incident jusqu'au fleuve. Arrivé au fleuve, ils nous ont dit:

- Maintenant on traverse mais vous pendant votre traversée il faut que Eugène s’applique à écoper. Surtout, pendant qu'il écope, qu'il fasse attention à ne pas écoper près du 
calfeutrage pour ne pas le sortir. N'arrache pas le calfeutrage pour ensuite sombrer. Quand tu écopes, il faut que tu ailles vers l'arrière.

Il a donc fallu que moi je m'asseye tout à l'arrière, sur le rebord de la poupe de la pirogue. J'étais donc perché et Eugène a reculé pratiquement à la place où j'aurais dû être assis. Et comme la proue de la pirogue était un peu en l'air, l'eau, dès qu'elle entrait, venait là, à l'arrière. Ainsi il écopait rapidement. On a écopé, écopé... jusqu'à notre arrivée au lieu-dit Mkalayeka l'Ancien, au village de Mloka.

Kina Serge na mwenzake Thomas wakabakiwa na kafi mbili nao. Wakawa wanavusha na wale anavusha mtu mmoja. Mimi sasa nilikuwa 'navusha,Eugène akawa anatoa maji yanayoingia kidogo kidogo. Akawa anatoa, yaani tukavusha kutoka hapa tulipopata tukio mpaka kupata mto mkubwa, yaani inaweza kuwa kama meta hamsini. Umeona, tukavusha mpaka tukapata mto mkubwa. Tulipopata mto mkubwa pale tukaambiwa :

- Sasa hapa tunakata lakini sasa nyinyi ukataji wenu uvushaji wenu lazima sasa Eugène ujitahidi sana kutoa maji. Na maji yako unatoa angalia usije kutoa karibu na hayo matambaa ukaja kutoa matambaa yenyewe wakati unakupamaji. Usije ukatoa matambaa yenyewe mkazama. Kuwa maana hiyo ukitaka kutoa maji lazima urudi nyuma.

Sasa kule nyuma kidogo kulikuwepo hakujapasuka mpaka nyuma. Ikabidi mimi nikakaa nyuma ya ngalawa kabisa juu kabisa ya magudi tuseme. Nikakaa juu, yule Eugène akasogea karibu na pale anapokaaga dereva, akawa sasa maji yote yanapoingia yanakimbilia kule kule nyuma. Kwa sababu mbele ngalawa iliinuka. Sasa akawa anatoa maji kwa wepesi. Toatoa maji sisiiii... mpaka tukafika kwa mzee Mkalayeka, hapo Mloka panaitwa hapo.

On a abordé. Après avoir abordé, il n'y avait qu'une chose à faire. On s'est dit que notre pirogue était hors d'usage. Il fallait d'abord qu'on la hâle sur la terre ferme. Si on l'avait laissé à l'eau, elle aurait sombré. Ils nous ont aidés à sortir la pirogue et à débarquer le poisson, mais en fait c'était de la chiquetaille. On 1'a sortie de l'eau et mise à sec. Que fallaitil faire ? Il n'y avait rien d'autre à faire que nos comparses aillent chercher notre armateur. Le groupe de Thomas, leur armateur c'était l'épouse du nôtre, elle s'appelait Mama Victor. Le groupe de Roger avait autofinancé son expédition. Ils avaient réuni l'argent, lui et Jacques, et ils s'étaient autofinancés. Leur poisson, ils le vendirent à Ignace, celui à qui nous voulions vendre nous-mêmes. Quand Ignace est arrivé on lui a dit : 
- Comme tu le vois on a eu des problèmes. Tu vas nous aider comment?

Il a répondu :

- Ah ! Par chance vous êtes revenus sains et saufs et la pirogue on pourra la réparer. Tout ça c'est une bénédiction. On organisera une autre expédition, vous irez pêcher et vous reviendrez ici, on négociera le poisson à un prix bon marché afin que je récupère la perte du crédit que je vous ai accordé.

Tukapaki pale. Tulipopaki ikawa hakuna jinsi. Tukasema hapa mpaka sasa hivi ngalawa yetu sisi imeshakuwa mbovu. Yaani sisi ngalawa yetu ndiyo iwe ya kwanza kuitoa hapa kuipeleka juu. Kwa sababu tukisema tuiache hapa itazama tena. Wakatusaidia ile ngalawa kuitoa pale na samaki vile vile na makasamala wale, samaki wenyewe makasamala !!! Tukaitoa kwenye maji tukaiweka gongoni. Cha kufanya nini ? Hakuna cha kufanya.

- Mfuateni tajiri !

Wakamfuata tajiri wale wenzetu, wale kina Thomas Ngwale. Tajiri yao alikuwa ni yeye huyo lakini alikuwa mkewe anaitwa Mama Saidi. Ndiyo alikuwa tajiri yao Majuta tajiri yao, yaani wao walijisafirisha wenyewe. Walichangachanga hela yeye na Jacques wakajisafirisha. Samaki waliowapata wakamwuzia Mzee Ignace tuliyetaka kumwuzia sisi, wakamwuzia wao. Mzee Ignace kufika pale tukamwambia :

- Sisi bwana tumeshapata msukosuko kama huu. Sasa itakuwaje ?

Akasema :

- Aaah ! Bahati nyinyi wenyewe mmerudi salama na chombo tunaweza kukifanyia ukarabati kikawa kizima. Yote ni heri. Tutapanga kazi tena tripu nyingine. Mtaenda kuvua mtarudi hapa, ila tutauziana kwa bei nafuu ili mradi mimi nifidie pale deni langu liliyopotea.

On lui a :

- D'accord, mais nous, on fait comment maintenant ? Comment on est, on n'a même pas de quoi payer notre retour à la maison et à la maison on ne sait même pas ce que nos enfants vont manger!

Qu'est qu'Ignace a fait?

- D’abord, votre chiquetaille de poisson je vais l'acheter, mais pour un bas prix. 
On a dit d'accord. Les poissons qu'on a vendus et qui étaient en morceaux, il y en avait 700. Après l'avoir vendu, on a obtenu 30000 shillings (15 euros)...

- De ces 30000 shillings, je ne prélèverai rien pour rembourser l'avance que je vous ai faite, même pas 10 shillings ! Je vous laisse ces 30000 shillings que vous vous les partagiez tous les deux.

J'ai pris 15000 shillings et mon comparse a pris 15000 shillings. A Mloka, chez ma sœur où je restais, j'ai donné à peu près 7000 shillings. C'était comme une sorte d'épargne parce que lorsqu'on pêche dans le Selous, ce n'est pas permis, c'est du braconnage. Il se peut que les gardes vous poursuivent et quand vous rentrez à Mloka, l'armateur peut vous dire qu'il n'a pas d'argent.

Tukamwambia :

- Sawa ! Sasa itakuwaje ? Hapa tulipo hatuna hata nauli ya kurudi nyumbani, na nyumbani hatutambui watoto zetu mpaka sasa hivi watakula nini.

Mzee Ignace akafanyaje ?

- Basi cha msingi mimi haya makasamala yenu nitayanunua mimi. Lakini kwa bei iliyo nafuu.

Tukasema sawa. Samaki tulipouza wale makasamala walifika makasamala kama mia saba, kwa maana hiyo baada ya kuuzauza samaki tukapata shilingi elfu thelathini umeona. Kupata shilingi elfu thelathini akasema :

- Shilingi elfu thelathini hii hapa sitakata deni hata shilingi kumi, "nakupeni yote elfu thelathini nyie wawili mgawane.

Mimi nikachukua shilingi elfu kumi na tano na mwenzangu nikampa shilingi elfu kumi na tano. Mimi nyumbani pale pale Mloka kwa dada yangu nilimwachia kama shilingi elfu saba. Yaani ilikuwa kama vile balanzi maana kwa sababu tunaenda kule, hatuendi kwa kibali tunaenda kwa kuiba. Maana unaenda kule wenda magemu wanaweza kukutimua. Ukirudi pale tajiri mwenyewe anaweza kukuambia :

- Mimi sasa hivi hela yenyewe sina na nini. 
Alors ces 7000 shillings, ils servent à quoi ? A te payer ton retour à la maison. Par chance, nous n'avons pas été poursuivis par les gardes mais nous avons eu un problème avec l'hippopotame. Donc, une fois de retour à Mloka et après que l'armateur eût acheté le poisson, nous n'y avions plus rien à faire. Il nous a dit :

- Rentrez chez vous mais ne traînez pas ! Revenez pour réparer la pirogue et pour repartir pêcher. Dieu prendra soin de vous.

Quand on est partis de là-bas, nos compagnons nous ont donné un peu d'argent parce que nous formions une sorte de coopérative. Ça signifie que si l'un d'entre vous a dû s'enfuir, alors tous ceux qui ont participé à l'expédition, une fois de retour, comme untel a été poursuivi par les gardes, celui qui a de l'argent lui donne 1000 ou 2000 shillings. Ou encore quelqu'un a été éperonné par une souche la nuit, celui qui revient avec du poisson, en arrivant à la maison, il lui donne même 3000 ou 5000 shillings. Nos compères, ils nous ont donné chacun 1000 shillings. On avait donc 4000 shillings (chacun). Avec ces 4000 shillings et l'argent que nous avions, on avait de quoi retourner à la maison et nourrir nos enfants. Nous avons traîné un peu et Roger est arrivé à Kipo avant nous.

Ikabidi elfu saba yangu itakuwa nini ? Nauli ya kurudi nyumbani. Bahati nzuri lakini magemu hawajatutimua ndiyo tumepata msukosuko wa 'boko. Tulipofika mpaka pale tajiri ndiyo amenunua samaki, tayari, sisi tukawa tumebaki pale hatuna la kufanya. Akasema :

- Nyie nendeni kwanza nyumbani lakini msichelewe, njooni tena, tuikarabati ngalawa, nendeni tena. Mungu anaweza kujaalia.

Sawa sasa mimi tulivyotoka pale wenzetu vilevile wakatupa kidogo hela kwa sababu imekuwa kama vile chama. Ina maana inaweza mtu akawa amekurupushwa basi nyie wote mlioenda kule mkirudi pale, jamani fulani hapa amefukuzwa na magemu basi mtu mwenye shilingi elfu moja, elfu mbili 'nampa. Mtu pengine kaliingia gogo usiku anarudi na samaki wake kwa hiyo tukifika nyumbani mtu anampa hata elfu tatu, tano. Sasa sisi wenzetu walitupa elfu, elfu. Kwa hiyo sisi tulipata shilingi elfu nne. Mimi nikapata shilingi elfu mbili na mwenzangu elfu mbili. Kwa maana tulikuwa na shilingi elfu nne. Elfu nne tukichukua na hela zetu kidogo zile, tukawa tumeshapata nauli ya kurudi nyumbani, kuja kuwapa watoto zetu nyumbani huku chakula. Tukarudi nyumbani, tulivyorudi nyumbani huku siku, siku ile tulivyorudi ile sisi hatukuwahi kurudi. Roger akawahi kurudi nyumbani. 
Il les a informés que nous étions bien rentrés mais que Maurice et Eugène avaient été attaqués par un hippopotame mais qu'ils s'en étaient bien sortis.

- Je les ai laissés à Mloka mais je pense qu'ils arriveront demain !

Effectivement, nous sommes arrivés le lendemain. On est resté un peu au village et ensuite il a fallu retourner à Mloka, moi et mon cadet. Arrivés chez notre armateur, il nous a dit qu'il n'avait pas d'argent.

- Peut-être que j'aurais une rentrée d'argent dans quatre jours et je vous donnerais alors du travail, vous pourrez repartir.

C'est alors qu'un type appelé Le Chauve est intervenu. C'est le cadet de Bernard, il est de ma belle-famille. Il m'a dit :

- Beau-frère, je vais financer votre expédition, qu'est-ce que tu en dis?

Je lui ai dit :

- D'accord, mais as-tu une pirogue?

Je lui ai dit que ma pirogue était endommagée, qu'il fallait la réparer.

- Quel genre de réparation?

Je lui ai dit qu'on avait besoin de clous et de tôle ou de plaques de plastiques

Akaja kuwapa taarifa kuwa :

Sisi tumerudi salama ila kina Mudi na mwenzake Eugène wamepigwa na 'boko lakini wote ni salama. Mimi nimewaacha Mloka labda watu hawa kufika hapa kesho ndiyo watafika hapa. Sisi ndiyo kesho yake tukafika hapa. Kukaa hapa tukabidi tena tuondoke mimi na bwana mdogo. Mimi na bwana mdogo tukaondoka hapa mpaka tukafika Mloka tena, kwenda kwa tajiri yetu, akatuambia :

- Mimi sasa hivi hela sina. Labda msubiri kama siku nne mbele, ndiyo inaweza kuagiziwa hela, nikakupangieni tena kazi, ndiyo mkaenda.

Akatokea jamaa mmoja anaitwa Le Chauve. Yaani mdogo wake Bernard yule. Kwa mimi yule shemeji yangu, akaniambia :

- Shemeji sasa mimi itabidi nikusafirishe, unasemaje?

Unaona? Nikamwambia :

- Sawa unisafirishe !

- Ngalawa unayo? 
Nikamwambia :

- Mie ngalawa yangu ninayo lakini mbovu. Ngalawa inahitaji matengenezo.

- Matengenezo yenyewe vipi ?

Nikamwambia :

- Tupate misumari na bati au maplastiki tukaigongee.

- Combien de clous pour que la pirogue ne prenne plus l'eau que vous puissiez repartir?

- Ah! Pas de problème.

Il a mis la main à la poche et il en a sorti 1000 shillings pour acheter des clous d'un quart de pouce. On a acheté les clous, on a réparé la pirogue, jusqu'à ce qu'elle soit bien et qu'elle ne prenne plus l'eau. La pirogue était en bon état. Il est venu.

- Ça y est, la pirogue est réparée !

Il nous a donné du travail. Il nous a acheté des filets, il nous a donné une avance pour partir en brousseet une avance pour la famille. En tout Le Chauve nous avait avancé 38000 shillings pour l'expédition. Comme l'avance faisait 38000 shillings, il nous a dit :

- Qu'est-ce que tu dis de cette somme-là, beau-frère !

On lui a dit :

- C'est bon, il n'y a pas de problème.

On est restés là-bas un moment. Il nous a donné l'argent tel jour et on est restés trois ou quatre jours avant de partir en brousse. Il y avait Eugène et moi et Duchemin accompagné d'Aurélien. Il y avait aussi un autre type appelé Jumeau-Terrible. Il était accompagné de Jean L'Habitant et de beaucoup d'autres... Nous étions pratiquement à 10 pirogues le jour du départ.

- Misumari mingapi ili ngalawa isitowe maji, tupate kuendea. Aaah ! Hilo siyo tatizo.

Ikabidi akaingiza mfukoni akatoa shilingi elfu moja kwanza kuenda kununua misumari ya robo inchi. Tukanunua misumari, tukaenda kule tukaigongee ngalawa yetu ile, mpaka ngalawa ikawa safi, haitoi maji wala nini. Ngalawa ikawepo nzima. Akaja pale, haya tayari ngalawa ishakuwa nzima. Akatupangia kazi. Akatununulia nyavu, akatupa posho ya 
kuondokea porini, akatupa na posho ya kuacha majumbani kwetu. Safari yetu kwa jumla tuliyopanga ilikuwa shilingi elfu thelathini na nane kwa Le Chauve. Ilipofika elfu thelathini na nane akatuambia hela hiyo unasemaje shemeji, tukamwambia aaha, hapa mie nipo shwari hamna tatizo. Tukakaa paleeee, alitupa hela leo tukakaa kama siku tatu ya nne tukaondoka porini, tulipoondoka porini, ndiyo nikawa mimi na Eugène, na Duchemin alikuwa na Aurelien. Halafu alikuwa mwengine anaitwa Jumeau-Terrible. Alikuwa na Jean L'Habitant na watu wengine wengi tulikuwa karibu ngalawa kumi tulipokuwa pale, siku hiyo tulipotoka pale tukaondoka.

Nous ne sommes pas passés par le fleuve mais par l'un de ses chenaux. En saison des pluies, il y a de nombreux chenaux qu'on peut emprunter et donc nous sommes partis aux environs de 5 heures du matin et nous sommes passés en plein jour tout simplement. On est arrivé à un endroit appelé Mvumo Mmoja, très en amont, et on a dormi là jusqu'à un peu avant 16 heures. Il a fallu ensuite traverser le fleuve. Ce n'est pas facile de traverser le fleuve en plein jour parce qu'on braconne et qu'on peut rencontrer les gardes en bateau à moteur. Nous on est en pirogue et eux sont en bateau à moteur, ça fait une différence ! On a traversé un peu avant 17 heures jusqu'à atteindre le lac Manze. On a pêché, on est resté six jours durant. On était dans la brousse, on a pêché, on a attrapé du poisson, on est repartis. On est revenu à Mloka et cette fois-ci on n'a pas eu d'ennui, ni avec un hippopotame ni avec quoi que ce soit. Une fois arrivés là-bas, on a vendu notre poisson à Le Chauve et il nous a donné notre argent. Après qu'il nous a donné notre argent, on est retourné à la maison. Depuis que je suis revenu à la maison, je n'ai pas encore eu l'occasion d'y retourner. Mon enfant était malade et je m'occupais de lui. Ensuite est venue la saison des semis.

Siku lakini tulivyoondoka hatujapita mto wa ndani tukapita mto wa nje. Kwa sababu kipindi cha masika inakuwa mito mingi ya kupita, kwa maana hiyo pale tukatoka kama majira ya saa kumi na moja asubuhi, tukawa tunapita mchana, tukapita mchana tuu. Mpaka tukafika sehemu moja inaitwa Mvumo Mmoja. Juu kabisa huko tukafika kule, tukalala kule. Mpaka majira ya saa kumi kasoro ya usiku. Ikabidi sasa tukakata bahari, mto mkubwa, yaani mto huo mkubwa kukata mchana si rahisi kwa sababu tunatumia kuibaiba kwa maana hiyo mchana inawezekana ukaonana na wenyewe wanapita na boti tu. Sasa sisi tunatumia ngalawa na wao wanatumia boti inakuwa tofauti. Tukakata majira ya saa kumi kasoro mpaka tukafika bwawani kwetu Manze kule. Tunavuavua, tukakaa kule kama siku sita. Tukakaa porini, 
tukavua tukapata samaki, tukarudi. Tulivyorudi mpaka niniye (?) tukafika Mloka sisi, tripu hii tukarudi salama hatujakutana na 'boko wala na kitu gani. Tulivyorudi mpaka pale, tukamuuzia Le Chauve samaki wale akatupa hela yetu. Alipotupa hela yetu pale mimi ikabidi nirudi mpaka nyumbani. Nilivyorudi nyumbani mimi tena sijapata bahati ya kwenda tena, mwanangu akawa anaumwa, sasa nikawa nipo kwa kumhudumia mwanangu. Nikamhudumia mwanangu tena, ikawa tayari kipindi cha mlao kupandapanda.

Il a fallu que je sème le maïs. Le maïs semé, je n'avais plus rien à faire. Où fallait-il que j'aille encore ? Il fallait que j'aille pêcher dans le Selous. Quand je suis revenu, je suis resté à la maison pour prendre soin de mes cultures. L'argent que j'avais obtenu là-bas (lors de la première expédition), je l'avais dépensé pour soigner mon enfant et quand je suis revenu (de la seconde expédition) j'ai eu de l'argent et alors j'ai pu rester à la maison. De juin jusqu'à septembre $^{8}$, je n’y suis pas retourné. Ensuite j’y suis retourné quatre fois. Mais cette fois-ci, c'était la saison sèche, ce n'était plus la saison des pluies. Il n'y avait plus qu'un seul passage, le fleuve lui-même. Mais Dieu a pris soin de nous. On y allait et on revenait, on y allait et on revenait... Et à présent je m'occupe aux travaux des champs, je cultive. Une fois terminés les travaux des champs, je me suis dit qu'il fallait que je me prépare à repartir. Mais il a plu et je n'ai pas pu partir. Je ne pouvais pas laisser mon épouse semer seule ${ }^{9}$, il fallait que je l'aide. Une fois les semis terminés, je n'avais plus de pirogue ! Je l'avais prêtée à Kassim Katanga et à Thomas Ngwale. Quand j’ai été les voir pour la récupérer, elle avait été volée !

Ikabidi sasa nianze kupanda mahindi ndiyo nikapandapanda mahindi, nilipomaliza kupanda ikawa sasa sina kazi ya kufanya. Ikabidi tena nitaenda tena wapi ? Selous kwenda kuvua nikavua, nilivyorudi nikabaki nyumbani tena kwa kuendelea kulima vizuri, kwa sababu ile hela yenyewe niliyopata kule yote nikawa nimemhudumia mwanangu, alipopona tena nikaenda tena niliporudi nikapata hela ndiyo nikakaa hapa nyumbani. Ndiyo sijaenda tangu mwezi wa sita sikuenda kule mpaka mwezi wa tisa. Ndiyo nikaanza tena kwenda tena kwa maana ndiyo kwenda tena mwezi wa tisa kule nikaenda kama tripu nne nilivyokwenda. Lakini kipindi hicho tena kilivyokwenda ilikuwa kiangazi sio masika ikawa kiangazi kwa maana hiyo mto unakuwa mmoja tu. Lakini Mungu akawa ametujaalia. Tukawa tunakwenda kule, tunarudi, tunakwenda, tunarudi. Mpaka sasa hivi mimi nipo hapa nikawa "nashugulika na

\footnotetext{
${ }^{8}$ Pour la saison de culture de décrue.

${ }^{9}$ Semis de la petite saison des pluies.
} 
mambo ya kilimo nilivyolimalima. Nilipomaliza kulima nikaona sasa nipange kuondoka. Mvua ikanyesha, kunyesha nikashindwa tena kuondoka, kwa sababu siwezi kumwacha mke wangu kupanda, itabidi nimsaidie kupanda. Nilipomsaidia kupanda kumaliza kupanda, kule nako ngalawa yangu ikawa haipo tena, nilimpa Kasim Katanga na Thomas Ngwale. Kwa maana hiyo ile ngalawa walivyoenda kule wao, wakaibiwa.

Maintenant je n'ai plus de pirogue et je devrais en louer une pour aller pêcher. Je ne supporte pas le désagrément de louer une pirogue. Je suis donc resté ici pour fouiller une nouvelle pirogue mais jusqu'à présent je ne l'ai pas encore fait. J'ai prévu de le faire, fouiller une pirogue, avoir ma pirogue à moi pour aller pêcher dans le Selous.

Jean-Luc PAUL : Tu la fouilles toi-même?

Non, je n'en suis pas capable. Je cherche un artisan. Je le paierai et je lui donnerai un coup de main.

Kwa maana hiyo mimi tena nikawa sina tena ngalawa.Nikienda kule mimi 'nakodi ngalawa "nakwenda kuvua. Sasa usumbufu wa kukodi ngalawa mpaka sasa hivi nikaona mimi haunitoshi, nikabaki hapa kwa kuhangaika kwa kuchonga ngalawa yangu, yaani mpaka sasa hivi bado sijaanza kuchonga ngalawa lakini nipo katika hatihati ya kuchonga ngalawa : kupata ngalawa na kwenda Selous.

Jean-Luc PAUL : Utachonga mwenyewe?

Hapana mimi mwenyewe siwezi kuchonga ila nitamtafuta fundi. Fundi nitamlipa hela tutakuwa tunashrikiana katika kuchonga.

\section{Témoignage 2, le 7 janvier 2008}

Avec Henri, on a voyagé au mois de décembre 2002. On s'est dirigé vers le lac Manze, dans la réserve du Selous. On s'est rendu là-bas et on a pêché beaucoup de poissons. La pêche terminée, nous étions au camp et nous avions déjà cuisiné. On s'est couché. Nous avions déjà 
fumé le poisson, on s'est allongé. Après s'être allongé, un avion est passé au-dessus du camp aux environs de 13 heures. Or, à ce moment-là, il y avait certains d'entre nous qui fumaient encore leur poisson. Malheureusement l'avion l'a vu et il a lancé un appel radio pour contacter ceux qui étaient en bateau sur le lac afin qu'ils nous poursuivent. L'avion tournait au-dessus de nous pour indiquer l'endroit. Les autres sont venus jusqu'au camp. Heureusement, ceux qui fumaient encore leur poisson alors que nous étions allongés, quand ils virent venir les gardes, ils nous ont réveillés. Ils venaient de nous réveiller quand les gardes commencèrent à tirer en l'air. Alors nous nous sommes rapidement dispersés. Eux, ils avaient repéré le camp et ils l'atteignirent et nous autres nous nous sommes enfuis. On s'est cachés pour les espionner. Ils sont arrivés au camp et ont détruit les fumoirs, ils ont pris les poissons et les ont chargés sur leur bateau. Ils sont montés à bord et puis ils sont partis.

Tulisafiri na Henri mwezi wa kumi na mbili mwaka elfu mbili na mbili. Tulisafiri kwa kuelekea bwawa la Manze, Selous. Tulikwenda kule tukavua samaki wetu wengi sana. Baada ya kumaliza kuvua tukawa kambini tumeshapika ugali tayari. Tumelala sasa. Tumeshamaliza kupiga moto samaki, tumelala. Baada ya kulala, majira ya saa saba mchana ikabidi ndege ipite eneo la kambi ile. Baada ya kupita kuna baadhi ya wenzetu wengine wakawa wanapigapiga moto samaki wao kwa muda huo. Bahati mbaya ile ndege ikaona. Baada ya kuona, ikapiga redio call wakawasiliana na wenye boti baharini. Ikabidi sisi tuanze kufuatiliwa. Ile ndege ikawa inazumguka juu kwa kuwaonyesha. Wale wakaja mpaka pale kambini. Walivyofika kambini bahati nzuri wenzetu wengine waliokuwa wanapigapiga moto samaki wao, sisi tulivyolala, bado walivyoona magemu wanakuja wakaanza kutuamsha hadi sisi wengine tulivyolala. Kutuamsha tayari magemu wakaanza kupigapiga risasi hewani. Kupiga piga risasi, sisi pale ikabidi tutawanyike wote kwa ghafla tu. Wale wakavizia mpaka kambini, wakafika, sisi wengine tukakimbia. Baada ya kukimbia, tukajificha kwa kuwachunguza. Wakafika mpaka pale wakavunjavunja uani zile, wakachukua samaki, wakapakia kwenye boti lao, wakawa wamepakia samaki tayari na wenyewe wamepakia, wamerudi.

Nous sommes revenus au camp. Eux, en venant, ils avaient sabordés les pirogues. Cela signifiait qu'on était coincé sur l'îlot, sans moyen de rejoindre le « continent ». On a attendu que tout le monde soit revenu pour se réunir et réfléchir ensemble à la manière d'atteindre l'autre bord. Une fois arrivés, les autres nous ayant rejoints, on s'est mis à réfléchir ensemble sur ce qu'il y avait lieu de faire. Comme il y avait des palmiers doum, on en a coupé 
beaucoup. On les a liés avec des cordes. Pour chaque équipage d'une pirogue, deux personnes, il y avait un tronc de palmier. On a mis (le radeau) à l'eau et on s'est assis dessus. Mais les palmiers ne se manœuvrent pas bien, ils vont accoster là-où ils le veulent bien. On s'est assis et on a traversé jusqu'à l'autre bord et Dieu nous a bénis. On s'est enfui ainsi et le radeau a été accoster sur le sable. Là où il avait bien voulu s'échouer, il y avait de l'eau jusqu'à la poitrine parce que c'est un très grand lac. Il nous a fallu tous descendre là. On a nagé, aux environs il y avait un petit chenal, on a nagé et Dieu nous a bénis. On a atteint la terre ferme et on a commencé le voyage de retour au village. Quand les gardes sont venus, c'était notre quatrième jour de pêche. On s'approchait du retour parce que cette fois-là on avait prévu de pêcher 5 jours. On a arrêté le quatrième jour, on a été chassés et on est repartis.

Baada ya kurudi na sisi tukafika hadi pale. Wale walivyofika pale ngalawa walizivunja. Ina maana tukawa kisiwani. Hatuna jinsi ya kutoka pale kwa kuelekea gongo lililokuwa moja, siyo la kisiwa. Tukangojana pale na wenzetu wote, wakatimia, akili tupange ushauri jinsi gani tutavuka kuelekea gongo moja. Tulivyofika pale, wenzetu walivyotimia tayari, tukashauriniana :

- Jamani tufanye vipi ?

Kwa kuwa kule kuna mivumo, ikabidi tukate mivumo, kwa wingi. Tukaifunga kamba. Kwa kila tundu moja la mivumo tumejaza kwa ngalawa tumepanda watu wawili wawili. Bahati nzuri tukatumbukiza baharini. Tukakaa tu mle mle. Kwa kweli mivumo haina uvushaji, ukae tu, inapotaka yeye kwenda kupaki ndiyo hapo hapo. Ikabidi tukae sisi, tumevuka mpaka ng'ambo, Mungu katuletea heri. Tumeporomoka hivyo hivyo, ikaenda kupaki mchangani tu ! Sehemu mpaka lilipotaka yeye limepaki maji ya kifua, kwa sababu ziwa kubwa sana. Wote ikabidi tushuke pale, tukaogelea kwa baadhi nyingine kulikuwa na kijito kidogodogo, tunaogelea, Mungu kaleta heri. Tumegeuka. Kugeuka na wenzetu wote wamegeuka, tukaanza safari ya kurudi kijijini. Tulikuwa na vuo la nne. Tunakaribia kurudi. Bado vuo letu moja tu, tuwahi kurudi. Kwa kuwa siku hiyo tulipanga tuvue mivuo mitano. Tumeishia la nne tu, tunafukuzwa, tukarudi.

Il y avait 9 pirogues de Kipo et de Nyaminywili, soit 18 pêcheurs. Les gardes ont détruit toutes les pirogues ! Il y avait aussi 2 gardiens du camp pour éviter que les poissons ne soient mangés par les hyènes. Il y a beaucoup de hyènes. Si tu laisses le camp sans surveillance, elles viennent. Il fallait deux gardiens. Au total, nous étions donc 20. Chaque équipage avait 
son propre armateur. Par exemple moi, j'avais autofinancé mon expédition. J'ai donc subi une grande perte et c'est pourquoi jusqu'aujourd'hui je n'embarquerai pas pour aller là-bas.

Jean-Luc PAUL : Et pour ceux qui avaient un armateur?

Si la cargaison de poissons d'un pêcheur est saisie, l'armateur qui le finance ne lui réclame rien, même pas un centime. Ce serait même un million, il ne réclamerait rien. Plus ! Quand le pêcheur rentre au village, son armateur doit lui donner ne serait-ce que 2 ou 3000 shillings pour qu'il puisse se nourrir. Il est doublement perdant car il doit prendre soin de son pêcheur.

Jean-Luc PAUL : Pourquoi pêcher à Manze qui est si loin ?

Il y a beaucoup de poissons et puis les interventions des gardes sont peu... Il n'y a pas beaucoup d'aller et venues de gardes, dans le temps en tous les cas. Mais maintenant, les gens n'y vont plus beaucoup parce que les gardes ont compris que les pêcheurs s'y rendaient nombreux.

Tulikuwa ngalawa tisa kutoka Kipo na Nyaminywili, yaani wavuvi kumi na nane. Wote wameivunja magemu. Tulikuwa na walinzi wawili vile vile, ili fisi wasile samaki. Kwa sababu kuna fisi wengi sana. Ukiacha kambi huru (bila kuilinda), wao wanakuja kula pale. Ikabidi tuwachukue walinzi wawili. Jumla tulikuwa $20 \ldots$ Kila mtu alikuwa na tajiri yake. Kwa mfano mimi mwenyewe nilijisafirisha mwenyewe. Kwa hiyo nimeingia short kubwa sana. Ina maana mpaka sasa hivi sitapanda tena ngalawa kwenda kule.

Jean-Luc PAUL : Na wale waliokuwa na tajiri ?

Kama mvuvi akitaifishwa, mzigo wake wa samaki, tajiri yake hatamdai hata senti moja. Ina maana hata kama milioni, hadaiwi. Na akirudi hapo hapo kijijini inabidi tajiri ampe angalau hela ya kula elfu mbili au tatu. Aingie short kwa mara ya pili, kumlinda yule mvuvi wake.

Jean-Luc PAUL : Sababu gani mnaenda mbali sana mpaka Manze kuliko mabwabwa ya karibuni?

Kuna samaki wengi, halafu purukushaniza za magemu... kidogo... zinakuwa... siyo pirikapirika nyingi za magemu, kama siku za nyuma... Lakini kwa sasa hivi, watu hawaelekei sana kwa sababu wameshagundua kwamba kule wavuvi wanakwenda sana. 
J'y ai pêché avec différentes personnes : Henri, Marcel, beaucoup de monde... Pascal...

Un fois rentré au village, les poissons que nous avons pêchés, mon compère me vend sa part et moi je vais vendre à un revendeur.

Jean-Luc PAUL : Pourquoi ton compère ne participe-t-il pas à l'autofinancement de l'expédition plutôt que de te vendre son poisson?

Ça dépend des objectifs de chacun. Certains, une fois qu'ils ont leur argent, ils n'ont pas l'intention de l'investir pour repartir pêcher. Ils ont peur de perdre leur argent, parce que c'est risqué. Ils préfèrent le dépenser. On arrive à s'entendre parce que le commerce c'est un contrat. Lui me vend à moi et moi je vends ailleurs à un autre prix. En revendant ainsi, j'obtiens un petit plus mais lui ne le voit pas parce qu'il n'est pas là quand je marchande avec le revendeur. Lui, il s'entend avec moi et moi je m'entends avec mon acheteur. A ce moment il n'est plus là. Par exemple, les poissons que Mgumba m'a vendu 350 shillings/pièce, je les ai revendus 380 shillings. Je n'ai jamais vendu mon poisson ailleurs qu'à Mloka. D'abord, je n'ai pas de licence pour transporter le poisson sur un autre marché. Et puis, autre chose, les gros marchands comme ceux à qui nous vendons ces jours-ci...

Nimevua na watu wa hali mbalimbali. Henri, Musa Sule, na watu wengi tu. Jumanne Mchawi...Wale samaki ninaopata kule, nikisharudi, yule mwenzangu ananiuzia mimi halafu mimi ninawauza hapa kwa mtajiri mwengine.

Jean-Luc PAUL: Kwa nini mwenzako anapendelea kukuuza samaki kuliko kujisafirisha?

Inategemea mtu malengo yake, mfano kama yeye hela akishaipata, basi hii hela hataipanga malengo ya kusafirisha tena. Anaogopa, kwa sababu sehemu yenyewe hatari, ile hela yake isije ikapotea. Bora atumie. Mwafaka unapita tu, kwa sababu biashara yote kuelewana. Yeye ananiuzia mimi, na mimi nauza sehemu nyingine kwa bei nyingine. Kwa maana hio ninavyouza kule mapato kidogo ninayaona, na yeye hashuhudii kwa sababu mimi naongea na tajiri mwengine na yeye hayupo. Yeye anapatana na mimi halafu mimi napatana na tajiri mwengine. Kipindi kile pale hayupo. Kwa mfano wale samaki nilionunua shilingi mia tatu na hamsini kwa Mgumbe nikawauza mia tatu na themanini. Sijawahi kuuza eneo lingine mbali lingine eneo ambalo ninauziaga. Ina maana kwa usafirishaji kwanza sijakata liseni ya usafirishaji kupeleka soko lingine.Na historia nyingine ina maana matajiri wakubwa wakubwa kwa mfano kama hao tunawauzia siku hizi...

Je veux dire que eux, les gros, une fois qu'on leur a vendu le poisson, quand on leur pose des questions sur le transport du poisson sur un autre marché, ils sont très prompts à te parler de sommes astronomiques. Ça nous décourage et on a jamais transporté, on ne comprend pas la procédure à suivre pour transporter le poisson sur un autre marché. 
Jean-Luc PAUL : Est-ce que tu pêches partout ou bien y-a-t-il des endroits que tu préfères ?

Pêcher au Selous c'est braconner. On va braconner avec la crainte des gardes. S'il y avait la possibilité de payer un permis de pêche comme ça se fait au lac Utunge wa Ngwenda, de mon point de vue, nous irions toujours pêcher dans le Selous. S'il y avait un permis de pêche pour le Selous, nous irions y pêcher. A cause des poursuites des gardes qu'on subit làbas, on est un peu craintifs. Parce que si tu finances une expédition un paquet d'argent, il se peut qu'on saisisse ta cargaison. Si on pouvait acheter un permis, il y a de l'argent à se faire là-bas, beaucoup d'argent... Si tu reviens sauf, tu as du poisson...Les poissons qui ont le meilleur débouché sont ceux du lac Suwanda. Ils sont très gros. A Nzelekela aussi ils sont très gros. A Manze, ils sont petits et nous y allons contraints, parce que le lac, jusqu'à récemment, n'était pas très surveillé. C'est pour ça qu'on y va pêcher.

Ina maana wale mabwana wakishatununulia samaki hapo hapo tukiwauliza mfano maswala ya usafirishaji kupeleka soko lingine wanakuwa watu wa mbio kweli kuhusu maswala ya usafirishaji, wanasema kiasi kikubwa sana. Sisi tunaganda hatujawahi kupeleka, hatuelewi utaratibu wa kupeleka kule samaki soko lingine.

Jean-Luc PAUL : Unavua popote au unapendelea sehemu zingine ?

Kwa shuguli za kiuvuvi kule Selous tunakwenda kwa wizi. Tunaenda kwa kuiba, kwa kuogopa magemu. Right ingekuwa tunakata kibali kule Selous, kama kibali wanachokatia kule Utunge (wa Ngwenda), kwa mimi nafsi yangu, tungeenda kuvua Selous. Ingekuwa ingepatikana kibali cha Selous, tungeenda kuvua Selous. Kutokana na purukushani zinazopatikana kule, tunakuwa waoga kidogo. Sababu mtu anapanga safari nyingine hela chungu nzima, unaenda kule unataifishwa. Ingekuwa unakata kibali, kule mapato yapo, mapato mengi. Ukirudi salama unapata samaki...Samaki wanavutia kibiashara bwawa la kwanza Suwanda. Wakubwa wakubwa samaki wake. Nzelekela, samaki wake wakubwa wakubwa. Manze samaki wake wadogo wadogo kibiashara, tunafanya hivo kwa tatizo. Kwa sababu lile bwawa kwa kipindi cha nyuma kilikuwa halina ulinzi mkali sana.Tumeenda kuvua hivo hivo.

Mais les lacs les plus attrayants du point de vue de la qualité commerciale des poissons, c'est Suwanda, Nzelekela, Tagalala et Utunge wa Selous. Là, les poissons sont très gros. Si tu 
y vas avec des mailles de 4 pouces, tu attrapes uniquement de gros poissons. Tu peux les vendre jusqu'à 700 shillings/pièce. Cependant ce lac est mal situé à cause des gardes qui ne s'en éloignent jamais. Ils restent là, près du lac... On craint donc ce lac parce qu'il a beaucoup de gardes. Les poissons sont gros et te remplissent de satisfaction. Le lac Utunge se tient au centre, il n'est pas loin du camp des gardes. Ils en font souvent le tour car ils sont proches. Ils viennent même à pied. Leur camp est à Kidahi. C'est le camp des gardes de Morogoro. Normalement, les gardes de ce camp patrouillent régulièrement à Utunge et à Tagalala ainsi qu'à Manze. Ils y viennent régulièrement. Les autres lacs, Suwanda, Nzelekela et aussi Manze, c'est un autre camp, celui de Temele. Ils ont la charge de 3 lacs. C'est seulement à Manze que viennent à la fois les gardes de Temele et ceux de Kidahi. Leurs patrouilles s'y rencontrent. Donc tu peux t'y faire poursuivre aussi bien par les gardes de Temele que par ceux de Kidahi. C'est souvent dans la journée qu'ils viennent patrouiller. Il y a des pêcheurs qui pêchent avec témérité, ils allument leur feu n'importe quand.

Lakini mabwawa yanayovutia kwa samaki wa biashara, Suwanda, Nzelekela pamoja na Tagalala, na Utunge. Samaki wake wakubwa wakubwa. Ukienda na numba 4 unavua samaki wakubwa tu. Unaweza ukauza mpaka shilingi mia saba. Samaki yake hasa Utunge wakubwa unauza shilingi mia saba. Isipokuwa bwawa lenyewe nalo limekaa vibaya sana kuhusu magemu, hawaendi mbali. Wapo pale pale bwawani. Kwa hio Utunge watu tunauogopa kwa sababu magemu wengi sana. Samaki wake wakubwa, wanaridhisha. Bwawa la Utunge limekaa center. Halipo mbali na kambi yao magemu. Wanazungukiazungukia mara kwa mara kwa sababu hawapo mbali. Wanatembea mpaka na miguu. Kambi yao ipo Kidahi. Ni kambi ya magemu wa Morogoro. Mfano magemu wa kule mara nyingi sana wanakuja Utunge na Tagalala, pamoja na Manze, wanakujaga siku moja moja. Mabwawa mengine ya Suwanda, Nzelekela pamoja na Manze kuna kambi nyingine ipo Temele. Hawa wanamiliki mabwawa matatu. Magemu wa Temele wanaenda mpaka Manze, na magemu wa Kidahi wanakuja mpaka Manze, wanakutana pale kwa patrol zao. Kwa hiyo unaweza ukakimbizwa na magemu wa Temele, unaweza ukakimbizwa na magemu wa Kidahi ! Kwa mara nyingi sana mabwawani mchana. Mchana mara nyingi sana wanakuja, wanachunguzachunguza. Wavuvi wengine wanavuaga na udharau, wanapiga moto wakati wowote.

Les gardes, dès qu'ils voient du feu durant leur patrouille, ils te pourchassent. Ou bien encore, un avion survole le camp et voit de la fumée, il fait un appel radio et les gardes 
viennent à ta poursuite. La nuit, les gardes se tiennent sur le lac. S'ils entendent que sur tel lac, par exemple le lac Suwanda, il y a beaucoup de pêcheurs et que chaque fois qu'ils s'y rendent en plein jour ils les manquent, alors ils organisent une patrouille de nuit et poursuivent les gens pareillement.

Des coups de feu, ils en tirent toujours. Certains tirent seulement pour t'effrayer mais à d'autres moments ils te visent carrément pour te tuer ! J'étais avec mon ami Henri, j'avais déjà fait souvent affaire avec lui et on s'entendait bien en affaires. On a été au lac Suwanda. On a pêché le premier jour, puis le deuxième jour. Le troisième jour, on s'est rendu sur le lac. Notre camp était sur un îlot. On avait relevé notre filet une première fois et on avait attrapé du poisson. On a été sur la berge pour démailler le poisson. On triait le poisson, on le démaillait. Malheureusement, les gardes étaient déjà là à nous observer pour voir où nous allions aborder. Dieu nous a protéger et nous avons abordé là où ils n'étaient pas. Ils nous auraient capturés. Mais Dieu nous a protégés, comme des saints. Arrivés sur la berge, là où ils n'étaient pas, ils ont démarré le moteur pour nous rejoindre. Ils venaient là où nous nous tenions et nous, nous nous sommes enfuis.

Wale wakishaona moto kwenye patrol zao, wanafuata. Aidha ndege imepita juu imeona Eugène, inapiga redio call, wanakuja magemu wanafuatilia. Usiku magemu na hawa wanakuwa mabwawani. Wakishasikia bwawa fulani, mfano bwawa la Suwanda, wavuvi wengi sana wanavua, kwa kila mchana wakienda wanawakosa, basi wanaamua kuweka doria yao usiku. Wanawakimbiza watu vile vile.Lisasi lazima wapige. Wengine wanapiga kwa kutisha tu, muda mwingine wanapiga kwa kukusudia kabisa ili wakuue !Nilikwepo na rafiki yangu Henri, kwa sababu nimeshafanya biashara naye sana, tulikuwa tunaelewana kibiashara. Tulikwenda bwawa la Suwanda. Kufika Suwanda kwenye uvuvi tumevua siku ya kwanza, tukaja kukavua tena siku ya pili. Siku ya tatu tunakwenda bwawani, kambi letu lilikuwa kisiwani. Tumepiga bigo (pigo) la kwanza la samaki. Tumepata, tukaenda pembeni, tunatoa wale samaki, tunachambua. Nyavu tunachambua, tunawatoa wale samaki. Bahati mbaya, wale magemu walishakuja mle, walikuwa wanatuangalia sisi, tutakwenda kuparki wapi ? Mungu analeta heri, sehemu ambapo tulienda kuparki toafauti na waliokuwa wao. Wangetukamata. Lakini Mungu akaleta heri, kama malaika tu ! Kufika pembeni ambayo sehemu wasiokuwa wao basi wale mabwana wakawasha boti lao kwa kutufuata pale tulipokuwa. Wakaja mpaka tulipokuwa, sisi ilibidi tukimbie. 
Comme nous nous étions enfuis, ils ont détruit notre pirogue. Si tu avais vu la manière dont nous étions vêtus, tu aurais eu de la peine. Je ne peux pas l'oublier. C'était des loques et nous étions déjà sur la terre ferme qui nous conduisait au village. Finalement, on n'avait tiré aucun profit et nos affaires étaient sur l'îlot. Nous n'avions pas le moyen d'y retourner pour aller chercher nos affaires. Après avoir été chassés, on est retournés au village par la terre ferme. On n'était pas encore arrivés, on rentrait tous les deux, moi et mon ami Henri, quand par malchance on a croisé les éléphants. Ils descendaient vers le lac pour s'abreuver et nous nous remontions vers Vikosi. On s'est mis à courir mais nous courrions vers d'autres éléphants ! Ils cassaient tout sur le passage et on les entendait venir. Il nous a fallu tous les deux monter sur un arbre. On est resté perchés 3 ou 4 heures, c'est une estimation, je ne sais pas exactement parce que nous n'avions pas de montre. On a laissé passer les éléphants jusqu'à ce qu'ils atteignent l'endroit dont nous venions (sur le bord du lac). Ils avaient tout piétiné sur leur passage, ils avaient beaucoup mangé et finalement ils ont été dans l'eau. Pendant qu'ils étaient dans l'eau, nous sommes descendus de l'arbre pour retourner au village. Heureusement, Dieu nous a protégé, on est arrivé jusqu'au village. On a demandé qu'on nous dépanne pour les vêtements. Des amis nous en ont donné et nous nous sommes habillés.

Baada ya kukimbia, ngalawa ile wakavunja.Sasa ukiangalia kwa nguo ambazo tulikuwa tulizivaa, inasikitisha sana. Siwezi nikasahau. Zipo ovyo ovyo halafu tupo gongo moja, ina maana la kutokea kijijini. Isipokuwa hatujaambulia kitu chochote kutokana na kambi yetu ipo kisiwani. Hatuna nyenzo nyingine ya kwenda pale, kufuatilia vitu vyetu. Sisi baada ya kukimbizwa tunapita gongoni tunarudi nyumbani. Kabla hatujafika nyumbani, tupo porini, tunarudi watu wawili tu, mimi na yeye rafiki yangu Henri. Bahati mbaya naye njiani tukakutana na tembo... Wanashuka. Wanakuja kunywa maji bwawani, sisi tunaelekea kule juu Vikosi. Pale cha kufanya tukaanza kukimbia, kule tunapokimbia na tembo wako kule kule. Wanavunja mbele yetu, tunawasikia. Ikabidi sisi tupande juu ya mti, wote wawili. Baada ya kupanda juu tumekaa karibuni masaa matatu au manne, inafika, kwa sababu tulikuwa hatuna saa. Tunatumia kukisia. Wale tembo tumewaacha mpaka wamefika lile eneo tulipokuwa sisi. Wanavunjavunja sana pale, wamekulakula sana, wakaenda mpaka kwenye maji. Wao wanakwenda kwenye maji na sisi tunashuka juu ya mti, tunarudi kijijini. Bahati nzuri, Mungu kaleta heri tumefika mpaka kijijini,tukaomba msaada wa nguo, sehemu zingine. Tukapata kwa marafiki zetu wengine, nguo tukavaa. 
Nous sommes rentrés à la maison. On avait rien pu sauver, nos affaires étaient restées sur l'îlot et il n'y avait pas d'autre pirogue pour aller les chercher. On a décidé de les y laisser. C'était en 2002, pendant la saison sèche.

Jean-Luc PAUL : Quelle est la période la plus dangereuse par rapport aux gardes?

En saison sèche car les touristes sont nombreux, on croit que c'est un bâteau de tourisme qui vient et finalement c'est le bateau des gardes. On a même peur d'aller voir quel bateau c'est, on suppose que c'est un bateau de tourisme et puis c'est bateau de gardes. On a peur d'aller voir car ils tirent des coups de feu. En saison des pluies, si tu entends un bateau, c'est nécessairement les gardes.

Désormais il ne va plus pêcher au Selous, il estime que c'est trop dangereux. On se fait poursuivre et tirer dessus par les gardes. Son expédition est autofinancée et il a tout perdu. Maintenant il cultive seulement et il a abandonné les autres activités. Avant, il ne voulait pas entendre parler de l'agriculture. Il continue à collecter du miel sauvage. A Utunge de Ngwenda, il irait bien pêcher si un armateur lui payait le permis.

Tunarudi nyumbani. Hakuna tulichoambulia, vitu vyetu vyote viko kisiwani na hakuna ngalawa nyingine ya kwenda kuvichukua vile vitu. Tukaamua kuviacha. Ilikuwa 2002, kilikuwa kiangazi.

Jean-Luc PAUL : Tukiangalia hatari za magemu, msimu gani umekuwa na hatari zaidi ?

Kiangazi kwa sababu watalii wanakuwa wengi. Unafikiri boti la watalii inakuja kumbe ni boti la magemu. Hata kwenda kuangalia ni boti gani tunaogopa. Unafikiri boti la watalii lakini ni la magemu. Tunaogopa kwenda kuangalia kwa sababu wanapiga lisasi. Lakini wakati wa masika, ukisikia boti lazima itakuwa boti la magemu.

\section{Témoignage 3, janvier 2008}

J'ai voyagé jusqu'au Selous avec Homer. Je suis allé au lac Suwanda. Le voyage a duré 2 jours. Une fois arrivés là-bas, nous sommes restés 4 jours (...)

Jean-Luc PAUL : Pourquoi avoir été en septembre au lac Suwanda et en décembre au lac Manze ? 
A cause de l'agressivité des crocodiles, on ne pêche qu'un seul type de poisson (pele : Citharinus congicus). Les nuits sans lune, tu ne peux pas pêcher à Suwanda où les crocodiles sont très agressifs. Ils peuvent mettre en pièces ton filet en une seule nuit. Une nuit sans lune les crocodiles sont très agressifs et déchirent beaucoup plus les filets que par une nuit au clair de lune. Par clair de lune, on les voit venir, on peut anticiper en les frappant avec la pagaie ou la perche. Ils laissent rapidement le filet. Mais dans l'obscurité c'est très difficile de s'en défaire, ils sont têtus. A Manze, il y a aussi beaucoup de crocodiles mais ils sont plus jeunes tandis qu'à Suwanda les crodociles sont très gros et sont capables de te causer beaucoup de dégâts. Les crocodiles de Suwanda sont trop dangereux, c'est pourquoi par nuit sombre on préfère aller pêcher à Manze où les crocodiles sont moins féroces, ils ne sont pas très grands. Il y a beaucoup plus de pêcheurs qui vont à Manze qu'à Suwanda. A Suwanda, tu n'y vas que par clair de lune. A Manze, que la nuit soit sans lune ou par clair de lune, tu t'y rends, tu travailles et il n'y a pas de problème.

Nilisafiri kwenda Selous na Homer (septembre 2007). Nilikwenda bwawa la Suwanda.Nilisafiri muda wa siku 2. Tulipofika kule tulikaa muda wa siku 4 (...)

Jean-Luc PAUL : Kwa nini mwezi wa tisa ulienda Suwanda halafu mwezi wa kumi na mbili ukaenda Manze?

Tunavua aina moja tu ya samaki kutokana na ukali wa mamba. Mwezi ukiwa gizani huwezi kuvua Suwanda. Suwanda kuna mamba wakali sana. Wanaweza kumaliza nyavu hata kwa siku moja. Mwezi ukiwa gizani mamba wanakuwa wakali zaidi na kula nyavu kwa sana kuliko kwenye mwezi. Kwenye mwezi wale wanaonekana jinsi wanavyokuja kule. Kwa maana hii unaweza kumuwahi kama unampiga pondo au 'bao. Wanaachia nyavu kwa haraka, lakini katika kiza inakuwa vigumu sana kuachia na wanakuwa wabishi. Manze kuna mamba wengi pia lakini wadogo kwa umri. Kwa sababu Suwanda ina mamba wakubwa sana, wenye uwezo wa kufanya hitilafu ya aina yote. Wale Suwanda mamba wamezidi, ndiyo maana huwezi ukiwa gizani sisi tunaenda kule Manze kwa sababu kule Manze mamba wanakuwa siyo wakali sana kama wale, kule hawajakomaa.Wavuvi wanaokwenda Manze ni wengi sana kuliko Suwanda. Suwanda unaweza kuenda na mwezi tu ! Lakini Manze mwezi ukiwepo unakwenda na giza ikiwepo tunafika, tunafanya kazi, haina tatizo.

Jean-Luc PAUL : Y a-t-il une différence de revenu entre les deux lacs? 
Il y a une différence de revenu car à Manze il y a beaucoup de poissons mais leur valeur marchande est faible. Le problème qui fait la différence c'est que les poissons de Manze sont légers, à Suwanda ils sont lourds. Et de même leur taille est différente. A Suwanda ils sont très gros. Un poisson fait 4 pouces ${ }^{10}$, mais 4 bons pouces ! Suwanda est proche. En trois heures tu sors du village (de Mloka) et tu es arrivé. Manze, il te faut la journée, 12 heures pour y arriver. Mais les gens s'accommodent de cet inconvénient parce que là-bas c'est un peu... même le bivouac est bon parce que... tu pêches comme braconnier... le bivouac est meilleur pour se cacher. A Suwanda, tu sais, s'il t'arrive quoi que ce soit, avec l'obscurité tu peux rencontrer du monde et tu n'as aucun moyen de t'échapper. A Suwanda, il n'y a pas de camp de gardes-chasse mais il y a des camps touristiques. Le lac est sévèrement surveillé, d'abord parce qu'il est proche et aussi parce qu'il est réputé pour ses gros poissons et qu'on peut s'y faire de l'argent. Avec 300 poissons, tu peux entendre dire qu'un type s'est fait 100 ou 200000 shillings tandis qu'à Manze je peux attraper même 500 poissons, jamais je n'aurais 100000 shillings !

Jean-Luc PAUL : Kimapato, kuna tofauti gani kati hayo mabwawa mawili?

Mapato yanakuwa tofauti kwa sababu Manze wanakuwa samaki wengi lakini si kibiashara (changement de cassette). Tatizo uzito umetofautiana. Wa Manze wepesi, Suwanda wazito sana. Halafu wana ukubwa tofauti vile vile. Suwanda wakubwa sana. Mmoja wa inchi nne lakini inakuwa nne kubwa!

Suwanda ni karibu sana. Unaweza kutoka pale kijijini (Mloka) kwenda kule na kutumia kama masaa matatu tu, umefika. Manze unatumia kucha, masaa 12 unafika kule. Lakini watu wanakubali usumbufu huu kutokana na kule kidogo... hata makazi yanakuwa mazuri kwa sababu... uvuvi unafanyika kule kama kiwizi... makazi yanakuwa mazuri kama ufichoni, Suwanda, kwa maana pale hamwezi hata kama inakutokea lolote, unaona, lakini na giza, unaweza kukutana na watu, ukashindwa kujitetea.

Suwanda kempu ya magemu hakuna ila kuna kempu ya wazungu. Ina ulinzi mkali kwa sababu ni bwawa la karibu kwanza halafu ni bwawa lenye sifa kutoa samaki wakubwa na mtu anaweza kupata hela. Samaki mia tatu unaweza kusikia mtu amepata laki moja au mbili, lakini Manze ninaweza kupata samaki hata mia tano, laki moja sipati !

\footnotetext{
${ }^{10}$ Ici, Thomas évoque maladroitement la taille de la maille du filet et non celle du poisson qui est bien supérieure à 4 pouces.
} 
Jean-Luc PAUL : Combien fait-il de voyages par an?

J'y vais deux fois par mois parce que si tu y vas cette fois-ci, il faut... D'abord parce que le voyage dure deux jours, puis tu pêches 5 jours, le huitième jour tu retournes. Ça signifie qu'une fois rendu à la maison, il faut que tu règles tes autres problèmes avant de retourner travailler là-bas. Et une fois de retour, tu dois te reposer. Tu t'appliques à bien dormir parce que tu as très sommeil. Là-bas le travail ne permet pas de bien dormir.

Jean-Luc PAUL : Est-ce que tu utilises ton filet une seule fois ou plusieurs expéditions $?$

Quelque fois, tu as de la chance et les crocodiles ne s'attaquent pas à ton filet. Il peut servir à nouveau. Mais il sera endommagé et il te faudra le réparer. Si beaucoup de crocodiles se manifestent, tu ne peux pas rentrer avec ton filet. Tu l'immerges sur place. A Manze, si tu es chanceux, tu rentres avec ton filet et tu peux le réutiliser deux fois. Mais même comme ça, c'est difficile de rentrer avec son filet parce que nous les pêcheurs quand on arrive là-bas on doit faire face au problème que les crocodiles déchirent ton filet. Donc, quand tu pars, tu laisses ton filet (à celui qui reste) pour qu'il continue à pêcher ce qu'il pourra avant qu'il ne se décide à partir. Ça m'est arrivé un paquet de fois !

Jean-Luc PAUL : Kwa mwaka unasafiri mara ngapi ?

Kwa mwezi ninakwenda mara mbili kwa sababu lazima ukienda mara hii... kwa sababu kwanza usafiri wake siku mbili unatembea, siku tano unaenda kufanya mavuvi, siku ya nane unarudi. Kwa maana ukisharudi nyumbani ushugulikie tena laana zingine za kuenda kufanya kazi kule. Na ukisharudi mpaka kufika hapa lazima upumzike tena. Unahitaji kulala kwa sababu una usingizi sana. Kwa sababu kazi za kule hazihitaji mlale wewe mara kwa mara..

Jean-Luc PAUL : Vipi, nyavu zinatumika safari moja tu au zaidi ?

Wakati mwingine unapata bahati, mamba hajakufikia kwenye nyavu ina uwezo wa kufanya kazi tena. Lakini zitakuwa zimepungua sana. Itabidi uiongeze kidogo. Kama wakitokea mamba wengi basi huwezi kurudi na nyavu, utaizamisha hapo hapo. Manze unarudi na nyavu unaweza kuitumia tena, bahati yako hata mara mbili unaweza kuzitumia. Lakini kunakuwa na ugumu sana kurudi na nyavu kwa sababu kawaida sisi wavuvi tunapofika kule tunaweza kupata hitilafu kwa mamba kula nyavu zako. Basi itabidi wewe nyavu zako umwachie wakati unapoondoka ili aendelee kuvua yeye kinachopatikana ndiyo ataamua kurudi. Ilishawahi kunitokea karibuni kibao. 
Cette expédition de Suwanda (dont je parlais), j'ai laissé mon filet à un collègue parce que le sien n'avait duré que deux jours après son arrivée. Après, il ne lui restait qu'un morceau d'environ 10 pieds sur $12 \ldots$ Huit flotteurs seulement ! Il est venu à notre bivouac et on a décidé de lui laisser notre filet. On le lui a laissé pour qu'il puisse au moins rembourser l'avance (de son armateur). Nous, on l'a aidé par solidarité entre pêcheurs. Un jour c'est lui et demain ça t'arrive à toi ! Lui, il pourra nous aider une prochaine fois. C'est comme une épargne que tu mets de côté.

Jean-Luc PAUL : Et si vous faites partie de la même expédition?

Si on s'accompagne lors d'une même expédition, il n'est plus seulement question de filet ! On coopère, lui et moi ne faisons qu'un. On pêche le poisson et quand il est fumé, on le partage, chacun charge le poisson dans sa propre pirogue et le rapporte à son armateur. Ce n'est pas seulement une question de filet ! S'il arrive que par malchance la pirogue soit heurtée par un hippopotame, qu'elle soit fendue, alors on s'entraide pour pêcher. On met le poisson dans une pirogue et le reste, ils le porteront sur la tête. Une fois rentrés, ils viennent prendre leur part de poisson dans notre pirogue et ils l'apportent à leur armateur. Ce qui compte, c'est qu'il rentre (sain et sauf) ! Qu'il fasse ses comptes là-bas et d'habitude tu l'aides pour qu'il ait assez d'argent pour manger.

Hata hii tripu ya Suwanda, nilimwachia nyavu mwenzangu kwa sababu alipofika pale nyavu zake alivua siku mbili tu ! Kisha ikawa nyavu imebaki kidogo tu, kama urefu wa futi 10 tu kwa 12. Maboya manne. Ikabidi aje kwenye kambi letu tukaamua kumwachia nyavu sisi. Tumwachie yeye akajikomboe na lile deni lake alilokuwa nalo. Sisi tunamsaidia kama mvuvi mwenzetu kwa sababu leo imetokea kwake kesho inaweza kutokea kwetu. Yeye anaweza kutusaidia siku za mbele. Ni kama akiba... Unaweka kiakiba kiubavuni.

Jean-Luc PAUL : Kama mpo katika safari moja ?

Kama tumefuatana naye kwa safari moja, yaani siyo nyavu tu... Yeye tumeshirikiana naye kimavuvi, mvuvi mwenziye mmoja na mimi nitatoka na huyu mmoja. Huwa tutavua samaki, tutakuja kugawa na wakati wakishakauka, kila mtu anapanga katika ngalawa yake anapeleka kwa tajiri wake. Na siyo nyavu tu, ikitokea na ngalawa bahati mbaya ukapigwa na 'boko, imechanika, tunasaidiana. Samaki tutatia kwenye ngalawa moja. Wanaobaki watapelekwa kichwani, wanapofika wanakuja kuchua samaki kwenye ngalawa yetu, atapeleka kwa tajiri yake. Lakini mradi naye arudi ! Akajue hesabu kule, na kawaida unamsaidia apate hela ya kula. 
Parce que quand tu es attaqué par un hippopotame, l'armateur ne peut pas dire qu'il a encore une créance. Il n'y a plus de dette parce que c'est quelque chose qui arrive couramment, on peut dire un mauvais coup du sort. Si l'armateur en a les moyens, il annule la dette. Même s’il s'agit de 10 pirogues dont la cargaison aurait été saisie par les gardes, même s'il s'agit de 20 cargaisons, il endosse la perte. Tous (les pêcheurs) n'ont plus de dette. Une fois que je pêchais dans le lac Utenge du Selous, j'ai été attaqué par un hippopotame. A Utunge, il y a beaucoup de crocodiles et ils sont gros. Je n'avais aucun secours, seulement Dieu. On a nagé jusqu'à la berge. La pirogue était fendue et il nous avait chavirés. On a été voir nos collègues, on leur a expliqué et il a fallu aller chercher la pirogue. Malheureusement le filet... on était sans... presque plus de filet, juste les ralingues. Les crocodiles avaient achevé le filet. Il a fallu que nos collègues nous aident à pêcher. On pêche ensemble et on partage comme ça. J'étais avec Aurélien, c'était en juin 2003.

Kwa sababu anapopigwa na 'boko tajiri hawezi kusema tena kwamba kuna deni. Inakuwa deni hakuna kwa sababu imeshatokea mwao, yaani bahati mbaya tuseme. Kama tajiri uwezo mwingine anao, anasamehe, yaani hata ikiwa ngalawa 10, anasamehe, kupigwa na 'boko, kutaifishwa mzigo wakichukua maaskari, hata mizigo ikiwa 20, za kwake (la perte). Wote hawana deni.

Niliwahi kupigwa na 'boko Utunge (du Selous) wakati nilikuwa 'navua. Utunge kuna mamba wengi na wakubwa. Nikawa sina msaada kunisaidia (ila) Mungu mwenyewe. Tukawa tumeogelea tumefika pembeni, lakini ngalawa alikuwa hakupasua, alitupindua tu. Tukaja pembeni, tukachukua wenzetu tukawelezea, ikabidi tukaenda kufuata ngalawa yetu kati lakini bahati mbaya ile nyavu... tulikuwa hatuna nyavu (hata) kidogo, tulibakia na milezi tu. Nyavu yote alimaliza mamba. Ilibidi wenzetu watusaidie tuvue pamoja, tukagawana vile vile. Nilikuwa na Hamisi Diunde. Ilikuwa mwezi wa sita mwaka elfu mbili na tatu. 
Cette histoire d'aller pêcher au Zaïre ${ }^{11}$, moi j'adorais aller y pêcher. Et ma vie durant, j'ai élevé mes enfants grâce au Zaïre. Mais je ne braconnais que du poisson, rien d'autre ! On allait jusqu'à Utunge du Selous, d'autres allaient à Tagalala, mais nous c'était Utunge. C'est l'endroit auquel j'étais habitué. On entrait sur le lac Utunge et on pêchait. Moi j'avais un don, si j'entendais quelque chose dans ma tête, si je rêvais de cette chose, alors sache que cette chose devait arriver.

- Lavallée, tu sais, aujourd'hui on va nous tomber dessus !

- Comment tu sais ça ?

J'ai dit :

- Moi, quand je rêve quelque chose, elle arrive ! Il faut que tu saches que c'est quelque chose qui va réellement arriver. Donc, aujourd'hui on ne pêche pas ! Et nos poissons, on les cache. Le filet aussi et on immerge la pirogue.

Lavallée a acquiescé en disant :

- D’accord grand-père, tu as bien parlé !

Nous sommes montés au-dessus du lac et nous sommes restés silencieux dans l'attente de ce qui allait arriver.

Historia hii ya kuvua uvuvi wa Zaire, mimi nilikuwa 'napenda sana kuvua Zaire. Na maisha yangu yote watoto, kulea nimelea Zaire. Na wizi wangu zaidi ilikuwa ni samaki tu ! Hakuna wizi wingine. Tunakwenda mpaka Utunge (wa Selous) :

- Wenzetu wanakwenda Tagalala kwanza sisi tuende Utunge.

Kule ndiyo nilikuzoea zaidi. Tunaingia Utunge, tunavua. Na mimi nilikuwa na kitu kimoja, kichwa changu kilikuwa kinaniambia chochote nikikiota basi ujue kitakuwa cha ukweli. Ninamwambia mwenzangu :

- Lavallée, bwana sasa mimi 'naona leo tutavamiwa hapa!

- Unajua je ?

Nikasema :

- Hapana, mimi nikiota kitu kinatoka hivi kuja hivi, basi ujue hicho kitu cha ukweli kabisa ! Sasa leo tuache kuvua ! Na samaki wetu tuwafiche hawa, na nyavu tufiche, na mtumbwi tuuzamishe.

Basi Lavallée ananikubalia kusema :

\footnotetext{
${ }^{11}$ Le Zaïre est le surnom donné au Selous.
} 
- Kweli kaka umezumgumza vizuri.

Sisi wenyewe tunapanda juu, tunakaa kimya, tunakaa kwenye njia ya matokeo.

Peu après, on a vu le garde qui se faufilait sur les berges. Il observait. J'ai dit à Lavallée

- Tu as vu le travail !

- Vrai!

- Bon, taisons-nous !

Les gardes sont restés silencieux sur la berge, ils se sont rejoints, ils étaient trois. Ils sont restés une heure. Ils observaient sans cesse. Ce jour-là, ils n'ont rien vu et ça a été pareil le deuxième et le troisième jour. Finalement ils sont partis. Alors on est revenus et on s'est mis au travail. On a pêché beaucoup de poissons, en plein jour ${ }^{12}$. Une seule jetée de filet ramenait 5 ou 600 poissons. Une fois qu'on les avait pêchés, on allait les sécher à environ un quart d'heure de marche du lac. On ne restait pas près du lac, on s'enfonçait en brousse là où on n'avait aucune chance d'être répérés... On a longtemps pêché de la sorte mais ceux qui viennent pêcher aujourd'hui, la nouvelle génération, ils n'ont pas de respect. Ils installent leur camp sur le bord même du lac. Quand les gardes arrivent et qu'ils voient ces camps, pour eux c'est une aubaine ! Ils ne perdent pas leur temps. Et les filets... ou pour écailler les poissons, ils écaillent sur la rive !

Mara tu tunamkuta yule gamu huyo. Anapita pembeni pembeni. Anatizamatizama ${ }^{13}$ namwambia Lavallée :

- Unaona hii kazi ?

- Kweli !

- Basi tunanyamaze !

Sasa gamu akawa anakaa pembeni kule kimya, wanakusanyika pale kama watatu watatu.. Basi wakakaa hapa muda wa saa mzima. Wakaangaliaangalia. Hawaoni kitu chochote siku ile mpaka siku ya pili, kimya, mpaka siku ya tatu. Wanaondoka tunakuja sisi nyuma tunafanya kazi. Basi hapo mchana tunaua vibaya samaki. Pigo moja mia tano, mia sita.

\footnotetext{
${ }^{12}$ Le narrateur précise «en plein jour» car c'est une pratique inhabituelle. Après le départ des gardes, les pêcheurs sont convaincus qu'ils sont à l'abri d'une nouvelle patrouille.

${ }^{13}$ Le verbe kutazama est souvent prononcé kutizama dans le Rufiji.
} 
Tunawavua wale tayari. Kuanika kwetu tunachukua baada ya kutoka pale kwenye bwawa mpaka huko tunaanikia, tuseme robo saa, tunavyotembea. Hapo sisi hatuko kule kule bwawani, lakini kule porini tunakoanikia hawezi atajua hata kidogo.

Sasa tumekwendakwenda, matokeo yake ikawa wanokuja kuvua sasa hivi, kizazi kipya hakina adabu. Wanaweka kambi mle mle pembeni ya bwawa. Magamu wakifika pale wakiona makambi yale, basi inakuwa raha. Hakuna magamu anayezidi kukaa sana. Halafu zile nyavu, nini, kwa kupalia samaki wanapaa pembeni.

C'est une honte. J'ai dit à Lavallée :

- La pêche est en train de mourir. Tu penses quoi de cette situation ? On a élevé nos enfants, ils ont étudié, ils ont fini l'école grâce au braconnage dans le Zaïre. Maintenant, le temps du braconnage est terminé. Tout ce qui reste c'est le danger. Aurait-on les moyens de s'enfuir ? Non ! Il faut porter le poisson, il faut aller jusqu'à Nkumbilo avec le poisson sur la tête. 270 poissons et personne pour t'aider à les charger sur la tête ! Si tu es fatigué, il te faut trouver un arbre sur lequel poser ton fardeau. Tu charges seul, personne pour t'aider à charger jusqu'à Mloka. Est qu'on peut supporter cette situation ${ }^{14}$ ?

Lavallée a dit :

- Moi, je ne suis pas prêt d'abandonner. Toi, tes enfants ont fini d'étudier, les miens pas encore.

- Si tu veux continuer, continue ! Je te souhaite bonne chance, continue...

Mes compères ont continué d'aller là-bas. Résultat, ils ont dit :

- Eh ! Roosevelt ${ }^{15}$, mon vieux, il est venu s'installer au-dessus du lac et maintenant ils tirent sur les gens !

Inakuwa hawa aibu. Ninamwambia huyu Lavallée :

- Sasa uvuvi unakufa. Unafikiri nini ? Hali hii ! Tumewalea watoto wetu, wamesoma, wamemaliza shule, kwa ajili ya wizi ya kuja Zaire. Sasa hivi wizi sasa hivi hakuna. Iliobakia sasa hatari. Una uwezo wwa kukimbia ? Uwezo hatuna ! Na samaki sisi tunabeba.

\footnotetext{
${ }^{14}$ Ici le narrateur décrit le retour, lorsque les gardes ont détruit les pirogues mais que le poisson a été sauvé et qu'il faut le rapporter à Mloka à pied.

${ }^{15}$ Le gérant d'une des nombreuses concessions de chasse du Selous, le nom a été changé.
} 
Tunakwenda mpaka Nkumbilo, kubeba samaki wa kichwani. Samaki 270 hakuna mtu wa kumtwisha mwenzake. Akichoka aweke kwenye mti. Ajitwishe, basi hakuna mtu tena ya kumtwisha mwenzake mpaka Mloka. Hali hii sasa hivi tutaweza?

Bwana Lavallée akasema :

- Mimi kweli bwana sitaachia karibu kwa sababu wewe mwenzangu umeshamaliza kusomesha watoto, mimi bado ninaendelea...

- Basi kama unaendelea, endelee wewe. 'bariki, endeleeni.

Wenzangu wakawa wanaendeleaendelea kule. Matokeo yake wakasema :

- Ah ah ! Patamisi bwana kaja juu. Patamisi kaja juu, sasa hivi wanachapa watu lisasi.

- Tu vois le travail ! Et là-bas à Vinangu, vous passez comment ? Naguère on passait sans problème à Vinangu mais à présent comment passez-vous ?

Il a dit :

- Vinangu, pour passer il faut que tu prennes le bateau à moteur.

- Aïe ! C'est nouveau ça ! Le bateau là-bas il est gardé. L'européen était un très chic type. Une fois, il nous a sorti d'affaire au beau milieu de la nuit. Les gens ont mal agi en lui volant son bateau. Qu'a-t-il fait de mal ? Maintenant, il a mis du monde pour surveiller son bateau. Et (en saison sèche) quand tous les bras du fleuve sont asséchés, on ne peut plus passer que par là. Mon cher Lavallée, moi je n'irai plus au Zaïre. Je reste ici.

Et je suis resté ici. Pêcher ? Je ne pêche plus rien. Je me suis demandé quel travail pouvait me procurer de l'argent et j'ai pensé à la réparation des récipients d'aluminium ou de plastique. Ceux qui sont brûlés ou fendus, je les colmate. C'est comme ça que je gagne ma croûte. Et puis je me suis dit que ce n'était pas suffisant. Il faut que j'aille cultiver pendant la décrue, des patates douces, des giraumons... J'ai traversé le fleuve (pour aller dans la plaine inondable). Notre travail : les patates douces et le giraumon.

- Unaona kazi hio ! Haya nyinyi pale Vinangu mnapita vipi ? Zamani Vinangu tulikuwa tunapita. Mnapita vipi ?

Akasema hapa :

- Vinangu sasa hivi mpaka ushike lile boti, ndiyo upite. 
- Aïe ! Mambo mapya hayo. Boti pale, pana mlinzi pale analinda ? Mzungu pale alikuwa nzuri sana. Katuokoa usiku sisi wa manane pale Hapo hapo watu walifanaya mabaya kumwibia boti lake. Ana ubaya gani yule ? Sasa hivi akaweka watu pale wa kulinda boti. Na barabara yote imeshakauka, maji yako pale pale (il n'y a pas d'autres bras d'eau pour passer, il faut passer par le fleuve car l'eau est basse). Bwana Lavallée, mimi siendi tena Zaire. Ninabaki hapa hapa.

Nikawa "nabaki hapa. Kuvua sivui chochote. Nikaona sasa mimi nifanye kazi gani ya kuweza kuingiza hela ? Nikaubuni ufundi wa kutengeneza misufuria na maplastiki. Yalioungua, yaliyopasuka, mimi 'nayaziba. Ikawa riziki zangu sasa hii ya kupata chochote. Nikaona hata haiwezekani, bora nivuke mto nikalime mlao viazi, maboga... Nikavuka mto kwenda ng'ambo ya pili. Kazi yetu kulima viazi na maboga.

On est resté là-bas et Dieu a pris soin de nous car j'ai été épargné par la tragédie du $\operatorname{lion}^{16}$. Benoît a été la première victime mais ça aurait dû être moi plutôt que lui que j'avais été secourir. J'aurais dû être le premier à être dévoré par le lion. Il a fallu déménager de la plaine inondable, aller sur l'autre rive et mon travail a été de réparer les marmites, les couteaux. Ça a été mon principal travail. Et puis j'ai attrapé cette hernie linguale (...)

Quelquefois tu voyages sans armateur. Quand tu vois qu'une expédition a été bonne, tu autofinances la suivante.

- Je paie moi-même les avances mon cher, n’avance rien. Je t'apporterai du poisson.

De quatre pirogues, je lui vends la cargaison d'une seule et je m'occupe de la commercialisation des autres. Tu vends alors au prix que tu fixes toi-même et les affaires sont bonnes $(\ldots)$

Nous pêchons la nuit. C'est la nuit que nous pêchons. On relève les filets quatre fois. Si tu ne te sens pas d'aller pêcher, tu écailles le poisson, tu le disposes sur les séchoirs et tu le fumes. D'autres surveillent les hyènes jusqu'à ce qu'on est fini d'allumer le feu. On retourne le poisson. On va pêcher à nouveau à 4 heures du matin.

Tukakaa kule, Mwenyezi Mungu akanijalia msukomsuko wa Simba, mimi umenikosa. Peneti ndiyo mtu wa kwanza, ndiyo nilikuwa mimi, kama kuliwa nilikuwa mtu wa kwanza

\footnotetext{
${ }^{16}$ Entre août 2002 et avril 2004, 35 personnes ont été dévorés par un lion mangeur d'hommes dans les villages du canton de Mkongo dont Kipo fait partie.
} 
kunidahi mimi badala huyu mtu niliyekwenda kumgombolea. Mimi nilikuwa mtu wa kwanza kuliwa na yule simba. Mpaka tulipokuja kuhama kuja ng'ambo hii, ikawa kazi yangu kutengeneza masufulia, visu, basi hiyo ilikuwa kazi yangu kubwa sana. Matokeo ikawa mimi sasa yakanipata malazi ya ngili ya hernia (...)

Unaenda mwenyewe bila kusafirishwa. Unajitegemea baada ya kuona, ah ! Safari hii kidogo mambo yangu mazuri :

- Posho yangu mwenyewe, bwana usininunue posho ya aina yoyote. Nitakuletea samaki.

Basi mitumbwi minne, "nampa mtumbwi moja, mtumbwi mitatu mnakalia mwenyewe. Unauza bei unayotaka wewe mwenyewe. Sasa mambo yangu yanakuwa mazuri. Uvuvi wetu sisi tunavua usiku. Usiku tunakwenda kuvua. Mapigo yetu manne. Hamu hatuna ? Tutatuta samaki na tutaanika, tutakwenda kupaa huko. Wengine wanalinda fisi, mpaka tunamaliza tunachoma moto. Kupindua piga moto tunapindua. Tunakwenda tena saa 10.

A 6 h30 on est de retour et on immerge nos pirogues, au fond. On va au camp, on écaille les poissons et on les dispose sur le séchoir sans allumer le feu. A midi précise, on sait que l'européen mange. A cette heure, il mange. On allume le feu mais c'est un petit feu... Papapapa... Ca y est, on 1'a déjà éteint. Et voilà comment on s'y prenait. La saison des pluies, tu bois du petit-lait. Tu n'as pas peur des gardes ou de quoi que ce soit, tu ne crains pas qu'ils laissent leur travail pour venir te chercher jusqu'ici. Mais durant la saison sèche c'est là que la pêche pose des problèmes. En saison sèche les eaux sont basses et il n'y a qu'un seul chemin pour passer. Les hippopotames sont regroupés. Il faut que tu passes au milieu d'eux et les gardes comprennent pourquoi les hippopotames se mettent à meugler. Et comme ils comprennent pourquoi les hippopotames crient, c'est inévitable, ils viennent. Ils savent qu'il y a du monde qui arrive. Il y a l'hippopotame et il y a aussi le ng'walala (Bostrychia hagedash). Le ng'walala ${ }^{17}$ nous pose énormément de problèmes. Il n'y a pas d'oiseau pire que celui-là, si on pouvait tous les tuer on le ferait ! C'est une véritable plaie et les gardes savent que des pêcheurs arrivent. Une fois on s'est fait attraper comme ça par un Européen.

Saa kumi na mbili na nusu tunarudi, na mitumbwi yetu yote tunazamisha. Chini. Sisi sasa tunakwenda pale tunawapaa samaki, tunawapanga ndani ya uani bila kuwachoma moto.

\footnotetext{
${ }^{17}$ Kwarara en kiswahili.
} 
Les Cahiers d'Afrique de l'Est

Saa sita balabala tunajua yule mzungu anakula. Saa hizi mzungu anakula. Piga moto, basi moto yetu unakuwa blablabla. Papapapapapa... Umeshazima. Basi ! Ndiyo tulikuwa tunatumia namna hio.

Masika kama chai na mkate. Hamna matatizo matatizo kuogopa gamu wala nini. Afanye kazi, aache kazi mpaka akukute wewe. Lakini hasa uvuvi uliokuwa wa tabu wa kiangazi. Wakiangazi maji yamesharudi, njia inakuwa moja. Sasa hio njia moja wale mpaka unakujua sisi wale boko wanakuwa pamoja. Lazima tupite pale pale kati ya boko. Sasa wale wanaelewa boko wanalilia nini ! Akielewa hivyo basi huyu boko analia hivo hukosi watu hawa wanakuja. Ndiyo maana wale mpaka wanakujua kwamba watu wanakuja. Kuna huyu boko na ng'walala. Huyu ng'walala anatuudhi sana! Hakuna ndege mbaya kama ingekuwa kuwaua ndege hawa wangekufa kabisa. Hawa wana... hasiri kubwa, mpaka wale wanajua kusema kwamba hawa sasa wavuvi wanakuja.

Tulikamatwa na mzungu.

On l'a supplié :

- Cher Européen, nous sommes seulement venus pêcher ici. On te demande de nous excuser, qu'on reste encore deux jours ou encore le temps que tu voudras.

L'européen a dit :

- Pas question! Vous ne resterez qu'un seul jour. Pêchez et rentrez chez vous. Ici c'est le Selous ! On travaille nous! On ne veut pas que les touristes vous voient. Vous effrayez les animaux. Vous entendez !?

- On a compris.

- Si vous n'avez pas de quoi manger, voilà ce que je vous donne. Tout le reste je vais le renverser. Je vous donne de quoi rentrer à Mloka, point final !

C'est comme ça qu'a fait l'Européen.

Lakini tulimwomba :

- Bwana mzungu we, sisi tumekuja kuvua huku. Hatufanyi mabaya mambo yetu sisi samaki tu hawa! Ndiyo sasa tunachokuomba, utusamehe hapa, tukae kwa muda wa siku 2, au vyovyote utakavyoamua wewe. 
Cette histoire d'aller pêcher au Zaïre ${ }^{11}$, moi j'adorais aller y pêcher. Et ma vie durant, j'ai élevé mes enfants grâce au Zaïre. Mais je ne braconnais que du poisson, rien d'autre ! On allait jusqu'à Utunge du Selous, d'autres allaient à Tagalala, mais nous c'était Utunge. C'est l'endroit auquel j'étais habitué. On entrait sur le lac Utunge et on pêchait. Moi j'avais un don, si j'entendais quelque chose dans ma tête, si je rêvais de cette chose, alors sache que cette chose devait arriver.

- Lavallée, tu sais, aujourd'hui on va nous tomber dessus !

- Comment tu sais ça?

J'ai dit :

- Moi, quand je rêve quelque chose, elle arrive ! Il faut que tu saches que c'est quelque chose qui va réellement arriver. Donc, aujourd'hui on ne pêche pas ! Et nos poissons, on les cache. Le filet aussi et on immerge la pirogue.

Lavallée a acquiescé en disant :

- D’accord grand-père, tu as bien parlé !

Nous sommes montés au-dessus du lac et nous sommes restés silencieux dans l'attente de ce qui allait arriver.

Historia hii ya kuvua uvuvi wa Zaire, mimi nilikuwa 'napenda sana kuvua Zaire. Na maisha yangu yote watoto, kulea nimelea Zaire. Na wizi wangu zaidi ilikuwa ni samaki tu ! Hakuna wizi wingine. Tunakwenda mpaka Utunge (wa Selous) :

- Wenzetu wanakwenda Tagalala kwanza sisi tuende Utunge.

Kule ndiyo nilikuzoea zaidi. Tunaingia Utunge, tunavua. Na mimi nilikuwa na kitu kimoja, kichwa changu kilikuwa kinaniambia chochote nikikiota basi ujue kitakuwa cha ukweli. Ninamwambia mwenzangu :

- Lavallée, bwana sasa mimi 'naona leo tutavamiwa hapa!

- Unajua je ?

Nikasema :

- Hapana, mimi nikiota kitu kinatoka hivi kuja hivi, basi ujue hicho kitu cha ukweli kabisa ! Sasa leo tuache kuvua ! Na samaki wetu tuwafiche hawa, na nyavu tufiche, na mtumbwi tuuzamishe.

Basi Lavallée ananikubalia kusema :

\footnotetext{
${ }^{11}$ Le Zaïre est le surnom donné au Selous.
} 
Mzungu akasema :

- Hapana, mtakaa siku zenu moja tu ! Mvue, mrudi nyumbani ! Hili sasa ni Selous. Tunafanya kazi sisi, sisi hatutaki watu kuwaonaona. Mnawafukuza wanyama. Mmenisikia ?

- Tumekusikia.

- Haya kama posho hamna, hii posho 'nakupeni. Hii nyingine yote, mimi 'nakwenda kumwaga. 'nakupeni posho ya kutoka hapa mpaka Mloka, basi !

Huyu mzungu akitumia hio...

\section{Bibliographie}

Paul Jean-Luc, Stéphanie Duvail, Olivier Hamerlynck. "Appropriation des ressources 'naturelles' et criminalisation des communautés paysannes. Le cas du Rufiji, Tanzanie". Civilisations (2011)/2:143-175

Paul Jean-Luc. "Les récits oraux luguru comme sources ethnographiques : enlèvement, poignade, meurtre et remplacement dans une population matrilinéaire de Tanzanie ». Les Cahiers d'Afrique de l'Est, n49 Varia, (2014) : 161-183 